\title{
Strong cosmic censorship in vacuum space-times with compact, locally homogeneous Cauchy surfaces
}

\author{
Piotr T. Chruściel*† \\ Alan D. Rendall ${ }^{\ddagger}$ \\ Max Planck Institut für Astrophysik \\ Karl Schwarzschild Strasse 1 \\ D 85740 Garching bei München
}

gr-qc/9410040

\begin{abstract}
We consider the question of strong cosmic censorship in spatially compact, spatially locally homogeneous vacuum models. We show in particular that strong cosmic censorship holds in Bianchi IX vacuum space-times with spherical spatial topology.
\end{abstract}

\section{Introduction}

It is widely believed that an important question in classical general relativity is that of strong cosmic censorship (SCC), due to Penrose [34]. A mathematical formulation thereof, essentially due to Moncrief and Eardley 31] ( $c f$. also [5, 6, 23]), is the following:

Consider the collection of initial data for, say, vacuum spacetimes, with the initial data surface $\Sigma$ being compact, or with the initial data $(\Sigma, \gamma, K)$ - asymptotically flat. For generic such data the maximal globally hyperbolic development thereof is inextendible円.

\footnotetext{
${ }^{*}$ Alexander von Humboldt fellow. On leave of absence from the Institute of Mathematics, Polish Academy of Sciences, Warsaw. Supported in part by a Polish Research Council grant KBN 2 P302 095 06. Present address: Département de Mathématiques, Faculté des Sciences, Parc de Grandmont, F 37200 Tours, France. e-mail: chrusciel@Univ-Tours.fr

${ }^{\dagger}$ Supported in part by the Federal Ministry of Science and Research, Austria.

${ }^{\ddagger}$ Present address: Institut des Hautes Etudes Scientifiques, 35 Route de Chartres, 91440 Bures sur Yvette, France. e-mail: rendall@ihes.fr

${ }^{1}$ When talking about extensions, unless explicitly specified otherwise we do not assume that the extension satisfies any field equations.
} 
The failure of the above would mean a serious lack of predictability of Einstein's equations, an unacceptable feature of a physical theory.

Because of the difficulty of the strong cosmic censorship problem, a full understanding of the issues which arise in this context seems to be completely out of reach at this stage. For this reason there is some interest in trying to understand that question under various restrictive hypotheses, e.g., under symmetry hypotheses. Such a program has been undertaken in [31], and some further results in the spatially compact case have been obtained in [9, 26, 5, 1]. Interestingly enough, the SCC issue remained open in the class of vacuum spatially compact, spatially homogeneous space-times because of the difficulties in understanding the global dynamical behaviour of the Bianchi IX models. The aim of this paper is to fill this gap. The first main result of our paper is the following:

Theorem 1.1 Strong cosmic censorship holds in the class of vacuum Bianchi IX space-times with $L(p, 1), p=1,2$ spatial topology.

It follows immediately from point 2 of Proposition 2.6, Section 2 below, that the result is wrong for spatial topology $L(p, 1), p>2$.

Recall that the standard way of proving SCC (cf. e.g. [9]) is to prove uniform curvature blow-up of the metric for all but a non-generic set of initial data. Now one expects this to be true for Bianchi IX models because of the apparently ergodic behaviour of the dynamics there, together with the uniform volume contraction as the "boundary" of the maximal globally hyperbolic development is approached (cf. e.g. [22] and references therein for

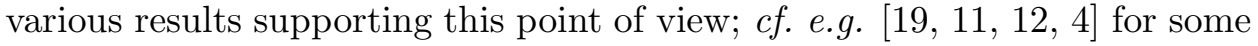
results suggesting that there might still be some surprises left on the road to the understanding of the dynamical behaviour of Bianchi IX models). Because of the complicated dynamical behaviour of the Bianchi IX models no rigorous proofs of curvature blow up have been published, and in fact it seems that no one has ever considered this question from a numerical point of view. Here we avoid this question by isolating directly those initial data which lead to the formation of a Cauchy horizon, leaving aside the problem of the long-time behaviour of the metric for those data which do not form a Cauchy horizon. This latter question has an interest of its own, and it certainly deserves further investigation. Fortunately, it is not needed in our proof of SCC for Bianchi IX models.

It should be pointed out that a version of Theorem 1.1 has been proved by Siklos in [42]. In that reference analyticity of the space-time is assumed. Moreover it is assumed there that the action of the isometry group on the globally hyperbolic part of the space-time analytically extends to the Cauchy horizon. The main work in this paper is to show that these hypotheses can be removed. We wish to acknowledge that in several places

\footnotetext{
${ }^{2} c f$. [10] for some results for Einstein - perfect fluid space-times and 36] for results on non-vacuum spacetimes with more general matter fields.
} 
we borrow heavily on the arguments presented in 42]. Some of the arguments here are perhaps somewhat simpler than the corresponding ones in [42; moreover we have found it necessary to reorganize Siklos' arguments in various places, because of different assumptions made. For those reasons we have found it useful to give a detailed exposition of the whole proof, which overall is rather similar to that of [42].

Recall that the issue of strong cosmic censorship arises because of possible non-uniqueness of solutions of the Einstein equations beyond Cauchy horizons. This is because any Cauchy data $(\Sigma, \gamma, K)$ define a unique (up to isometry) maximal globally hyperbolic development $(M, g)$, but whenever $(M, g)$ is extendible uniqueness of the vacuum extensions is lost, at least if one does not impose some further restrictions. In fact, in Section 5 we present a construction which shows that this occurs in any space-time with a Cauchy horizon, even when analyticity conditions are imposed on the extended space-time. The Taub-NUT space-times are a well known example with the property that every Taub-NUT initial data admit at least two maximal (vacuum) developments ( $c f$. [8]). These developments will be called the standard Taub-NUT space-times. Now the essential difference between those two space-times is in the way the boundaries $\partial \mathcal{D}(\Sigma)$ are "glued" to the globally hyperbolic Taub region, and it is natural to ask how many ways of doing this glueing exist. Our next result here shows that the standard Taub-NUT space-times exhaust all the possibilities:

Theorem 1.2 Let $(M, g)$ be a vacuum Bianchi IX space-time with a partial Cauchy surface $\Sigma$ such that $\mathcal{D}(\Sigma ; M)$ is maximal globally hyperbolic, with both the future Cauchy horizon $\mathcal{H}^{+}(\Sigma ; M)$ and the past Cauchy horizon $\mathcal{H}^{-}(\Sigma ; M)$ - connected, and $\mathcal{H}^{+}(\Sigma ; M) \cup \mathcal{H}^{-}(\Sigma ; M)$ - not empty. Then there exists a standard Taub-NUT space-time $(\hat{M}, \hat{g})$ and an isometric diffeomorphism $i$

$$
i: \stackrel{\circ}{\mathcal{D}}(\Sigma ; M) \rightarrow \stackrel{\circ}{\mathcal{D}}(i(\Sigma) ; \hat{M}),
$$

such that $i$ extends by continuity to a one-to-one map $\bar{\imath}$

$$
\bar{\imath}: \overline{\mathcal{D}(\Sigma ; M)} \rightarrow \overline{\mathcal{D}(i(\Sigma) ; \hat{M})} .
$$

Moreover $\bar{\imath}$ is a diffeomorphism of manifolds with boundary between $\overline{\mathcal{D}(\Sigma ; M)}$ and $\bar{\imath}(\overline{\mathcal{D}(\Sigma ; M)})$.

Here $\stackrel{\circ}{\mathcal{D}}(\Sigma ; M)$ denotes the interior of the domain of dependence $\mathcal{D}(\Sigma ; M)$ of a partial Cauchy surface $\Sigma$, while $\bar{\Omega}$ denotes the closure of a set $\Omega$.

In addition, in Section 5 we prove that the only maximal Taub-NUT spacetimes on which $G=S O(3)$ or $G=S U(2)$ acts by isometries are the standard Taub-NUT space-times, $c f$. Theorem 5.2. It would be of interest to find some other conditions, weaker than the above, which single out the standard Taub-NUT space-times in the collection of all the extensions of the globally hyperbolic region of the Taub-NUT space-times. 
Let us remark that in Theorem 1.2 the condition that each of $\mathcal{H}^{+}(\Sigma ; M)$ and $\mathcal{H}^{-}(\Sigma ; M)$ are connected is necessary. Indeed, counterexamples with non-connected, say, $\mathcal{H}^{+}(\Sigma ; M)$, can be constructed by making a "left future extension" for some set of null geodesics in Taub space-time, and a "right future extension" for another set of those. (The Cauchy horizon will of course not be compact here - clearly a compact, say, future, Cauchy horizon in a Bianchi IX space-time has to be connected.)

An interesting class of space-times in which to study the question of strong cosmic censorship is that of space-times evolving out of locally homogeneous initial data. Recall that a homogeneous geometry $(\Sigma, \gamma)$ is defined by the existence of a group $G$ which acts transitively on $\Sigma$ by isometries of $\gamma$. Loosely speaking, local homogeneity is then defined by the requirement of the existence of a locally transitive action by isometries, $c f$. the beginning of Section 1 for a precise definition. Recall that in the general relativists' terminology every space-time with locally homogeneous spacelike surfaces can be assigned a symmetry type, namely one of the 9 Bianchi types, and the Kantowski-Sachs $3^{3}$ type, cf. e.g. [27, 17, 40, 44, 24, 28]. It turns out that the methods needed to analyze the Bianchi IX case carry over without any essential modifications to the case of locally homogeneous initial data. Let us first mention the following result which shows that there could potentially be a SCC problem in the class of space-times with locally homogeneous Cauchy surfaces:

Proposition 1.3 1. For every symmetry type excep清 for Bianchi $V I_{0}$ there exists a spatially homogeneous vacuum space-time with a nonempty homogeneous Cauchy horizon.

2. Let the symmetry type be one of the following: Kantowski-Sachs, Bianchi I, II, III $=V I_{-1}, V I I_{0}$, VIII, IX. For every such symmetry type there exists a spatially locally homogeneous, spatially compact vacuum space-time with a non-empty locally homogeneous Cauchy horizon. No such space-times exist if the symmetry type is Bianchi $I V, V, V I_{h}$, $h \neq-1, V I I_{h}, h \neq 0$.

Note that the list of Bianchi geometries in the existence part of point 2 above coincides with the Bianchi A geometries, except for the type $\mathrm{VI}_{0}$ which is missing there. The non-existence part of the list for the spatially compact case consists of Bianchi B geometries, except for the type $\mathrm{III}=\mathrm{VI}_{-1}$ for which a spatially compact model exists.

As discussed at the beginning of Section 1 , strong cosmic censorship "halffails" in all locally homogeneous Kantowski-Sachs models. Indeed, those

\footnotetext{
${ }^{3}$ Following the standard terminology, the Kantowski-Sachs symmetry type is defined here by the requirement of local homogeneity together with the condition that there is no three dimensional isometry group acting transitively on the universal covering space. This implies that the universal cover is $\mathbf{R} \times S^{2}$, and the connected component of the identity of the isometry group of the universal cover is $\mathbf{R} \times S O(3)$ with the obvious action.

${ }^{4}$ We use the conventions of 42 for labelling the Bianchi groups.
} 
space-times always have a Cauchy horizon, say, to the future, and a curvature singularity to the past. Recall now that SCC is satisfied in homogeneous Bianchi I models (i.e., in Bianchi I models with globally defined Killing vectors; cf. e.g. [5] for a detailed discussion). Interestingly enough, it turns out that this is not the case any more when spatially locally homogeneous models with Bianchi I symmetry type are considered. In this class of spacetimes the SCC question turns out to depend upon the topology of the partial Cauchy surface. This is due to the fact, that some topologies allow only those locally homogeneous initial data for which the resulting maximal globally hyperbolic space-time is extendible. This is discussed in detail in Section 3 for the Bianchi I symmetry type. For the remaining Bianchi models in the discussion of SCC one needs to analyze case by case all the admissible topologies, as done in Section 3 in the Bianchi I case, which is a task which lies beyond the scope of this paper. (Such an analysis would be rather lengthy and tedious but probably otherwise straightforward, basing on the list of topologies given in [27].) Nevertheless, as we shall show below, there is a topology-independent sense in which SCC is satisfied in those models.

When considering the exact known solutions which contain Cauchy horizons, it is striking that they seem to display more symmetries than the typical representatives of the families they belong to. This is e.g. true for TaubNUT space-times, considered as members of the Bianchi IX family — for the former the isometry group is larger by a factor $U(1)$. This is true in some approximate sense in the class of polarized Gowdy space-times, in which one of the functions characterizing the solutions tends to a constant on each connected component of the Cauchy horizon [9]. Let us also mention the Isenberg-Moncrief conjecture [25], that space-times with a compact Cauchy horizon must have at least one Killing vector. As shown in Section \&, in a space-time with a locally homogeneous Cauchy surface for every point there exists a neighbourhood thereof on which a Lie algebra $\mathbf{g}$ of Killing vectors of dimension at least three is defined; $g$ will be called the local Killing algebra. [It turns out that $\mathbf{g}$ can be chosen so that it does not depend upon the choice of points.] In our context it is tempting to conjecture that $\mathbf{g}$ must be at least four dimensional whenever a Cauchy horizon occurs. This turns out to be true in the spatially compact case, and we have the following rather elegant formulation of SCC in this class of space-times:

Theorem 1.4 Let $(M, g)$ be a vacuum space-time with a compact locally homogeneous partial Cauchy surface $\Sigma$ such that $\mathcal{D}(\Sigma)$ is maximal globally hyperbolic and such that $\partial \mathcal{D}(\Sigma) \neq \emptyset$. Then the local Killing vector algebra is at least four-dimensional.

According to Siklos [42], there exist Bianchi VI $-1 / 9$ space-times with a Cauchy horizon for which $\mathbf{g}$ is only three dimensional This shows the necessity of the compactness condition above.

\footnotetext{
${ }^{5}$ S. Siklos, private communication.
} 
Let us emphasize that from what has been said in [42] it follows that a generic space-time (in the sense of parameter counting for simply connected models) of a given Bianchi type will not have four Killing vectors, so that Theorem 1.4 establishes indeed some kind of non-genericity of Cauchy horizons in this class of metrics.

This paper is organized as follows. In Section 2 we prove strong cosmic censorship in Bianchi IX space-times. In Section of we discuss the question of strong cosmic censorship in spatially locally homogeneous models with Bianchi I symmetry type. In Section 0 we consider general space-times with locally homogeneous, compact partial Cauchy surfaces, and we prove Proposition 1.3 and Theorem 1.4 there. In Section 5 we consider the question of uniqueness of extensions of the globally hyperbolic region of the TaubNUT space-times.

Acknowledgements We acknowledge the hospitality of the E. Schrödinger Insitute in Vienna during part of the work on this paper. P.T.C. also wishes to thank J. Ehlers and the Max Planck Institute for Astrophysics in Garching for hospitality. We are grateful to B. Edgar, G. Galloway, R. Geroch, G. Hall, J. Isenberg, S. Siklos, P. Tod and R. Wald for useful correspondence, or comments, or discussions.

\section{Bianchi IX space-times with Cauchy horizons}

In this section we shall prove Theorem 1.1. Let us start by fixing the terminology. $(M, g)$ will be called a vacuum space-time if $M$ is a four-dimensional manifold $[$, and $g$ is a Lorentzian metric on $M$ satisfying the vacuum Einstein equations (perhaps in a distributional sense). We shall moreover assume that $(M, g)$ is time oriented. Here, and everywhere else, we use the terminology of [21] unless indicated otherwise. Throughout this paper the symbol $\Sigma$ will denote a partial Cauchy surface in $M$, with $\gamma$ the metric induced from $g$ on $\Sigma$, and $K$ the extrinsic curvature tensor of $\Sigma$. $(\gamma, K)$ will always be assumed to satisfy the vacuum constraint equations.

Recall that given initial data $(\Sigma, \gamma, K)$ for the Einstein equations there exists (cf. e.g. [3]) a maximal globally hyperbolic development $(M, g)$ of $(\Sigma, \gamma, K)$. $(M, g)$ is uniquely defined by $(\Sigma, \gamma, K)$ up to isometry. We shall only consider here space-times which are maximal globally hyperbolic developments or extensions thereof. In the case of a space-time which is an extension of a maximal globally hyperbolic one with Cauchy surface $\Sigma$, we shall always assume that $\Sigma$ remains a partial Cauchy surface in the extended space-time. In particular $\Sigma$ will always be an achronal spacelike hypersurface. In our context this condition is equivalent to the condition of causal regularity of the extensions used in [8].

Let $\Sigma$ be a compact three-dimensional manifold and let $(\Sigma, \gamma, K)$ be initial

\footnotetext{
${ }^{6}$ All manifolds considered in this paper are assumed to be smooth, Hausdorff, paracompact, connected and orientable.
} 
data for the vacuum Einstein equations which are invariant under an effective action of $G=S U(2)$ or $G=S O(3)$, with three-dimensional principal orbits. A couple $(M, g)$ will be called a Bianchi IX vacuum space-time if $(M, g)$ is the maximal globally hyperbolic vacuum development of $(\Sigma, \gamma, K)$ or an extension thereof. (It follows from, e.g., 15 that $\Sigma$ must be a lens space $L(p, 1), p \in \mathbf{N}$; recall that $L(1,1)=S^{3}$ — the three-dimensional sphere.) Let $\stackrel{\circ}{\mathcal{D}}(\Sigma)$ denote the interior of the domain of dependence $\mathcal{D}(\Sigma)$ of $\Sigma$ in $(M, g)$; we shall write $\mathcal{D}(\Sigma ; M)$, etc. for $\mathcal{D}(\Sigma)$ when confusions are likely to occur. As has been shown in [5] (cf. also [0]) there exists a smooth action of $G$ on $\stackrel{\circ}{\mathcal{D}}(\Sigma)$ by isometries:

$$
G \ni g \mapsto \phi_{g}: \stackrel{\circ}{\mathcal{D}}(\Sigma) \rightarrow \stackrel{\circ}{\mathcal{D}}(\Sigma) .
$$

We have the following (recall that $f$ is in $C^{k, 1}$ if the $k$-th derivatives of $f$ are Lipschitz continuous; this will be the case if, e.g., $f$ is in $C^{k+1}$ ):

Proposition 2.1 Let $(M, g)$ be a vacuum Bianchi IX space-time with a metric $g$ of differentiability class $C^{k, 1}, k \geq 1$. Suppose moreover that $\partial \mathcal{D}(\Sigma) \neq \emptyset$, where $\partial \Omega$ denotes the topological boundary of a set $\Omega$. Then

1. There exists an open dense subset of $\partial \mathcal{D}(\Sigma)$ which is a submanifold of $M$ of $C^{k, 1}$ differentiability class.

2. There exist open sets $\mathcal{O} \subset \partial \mathcal{D}(\Sigma)$ and $\mathcal{U} \subset G$ such that for $g \in \mathcal{U}$ the maps $\phi_{g}$ extend by continuity to differentiable maps

$$
\hat{\phi}_{g}: \mathcal{O} \rightarrow \partial \mathcal{D}(\Sigma)
$$

with $\left\{\hat{\phi}_{g}(p), g \in \mathcal{U}\right\}-$ a three-dimensional subset of $M$.

Remark: Note that in general one does not expect a Cauchy horizon to be significantly better behaved than a Lipschitz hypersurface, even in spacetimes with a smooth metric. Here the higher differentiability of the (open and dense subset of the) Cauchy horizon follows from the existence of the isometry group. It should be noted that Proposition 4.4 below shows that $\mathcal{O}=\partial \mathcal{D}(\Sigma)$, which in turn implies that the whole $\partial \mathcal{D}(\Sigma)$ is a submanifold of $M$ of $C^{k, 1}$ differentiability class.

Proof: On $\stackrel{\circ}{\mathcal{D}}(\Sigma)$ the metric is smooth and there any Killing vector $X$ satisfies the equations

$$
\nabla_{\mu} \nabla_{\nu} X_{\sigma}=R_{\mu \nu \sigma}^{\lambda} X_{\lambda}
$$

As the metric on $M$ is $C^{1,1}, R_{\mu \nu \sigma}^{\lambda}$ is uniformly bounded on $\overline{\mathcal{D}(\Sigma)}$ in local coordinates, and from (2.1) it easily follows that $X$ extends by continuity to a vector field $\hat{X}$ of $C^{k, 1}$-up-to-boundary differentiability class on $\overline{\mathcal{D}(\Sigma)}$. Let $\mathbf{g}$ be the Lie algebra of $G$, by an abuse of notation we shall identify an element $e$ of $\mathbf{g}$ with the corresponding Killing vector field $\hat{X}=\hat{X}(e)$. 
Let $p \in \partial \mathcal{D}(\Sigma)$, there exists a neighbourhood $\mathcal{V}_{p}$ of the origin in $\mathbf{g}$ such that for all $g \in \mathcal{U}_{p} \equiv \exp \left(\mathcal{V}_{p}\right)$ we can define $\hat{\phi}_{g}(p)$ in the standard way, i.e., if $g=\exp (\hat{X})$, then $\hat{\phi}_{g}(p)$ is defined by following the orbit of $\hat{X}$ through $p$ a unit distance. Clearly $\hat{\phi}_{g}$ extends continuously $\phi_{g}$. Since $\phi_{g}$ maps $\stackrel{\circ}{\mathcal{D}}(\Sigma)$ into $\stackrel{\circ}{\mathcal{D}}(\Sigma)$, it follows that for $g \in \mathcal{U}_{p}$ the (locally defined) map $\hat{\phi}_{g}$ takes a neighbourhood $\mathcal{O}_{p} \subset \partial \mathcal{D}(\Sigma)$ of $p$ into $\partial \mathcal{D}(\Sigma)$. Because the Killing vectors are of differentiability class $C^{k, 1}$ up-to-boundary on $\overline{\mathcal{D}(\Sigma)}$, the maps $\hat{\phi}_{g}(p)$ are of differentiability class $C^{k, 1}$ with respect to $p$.

Let us show that there exists $p \in \partial \mathcal{D}(\Sigma)$ such that the set $\left\{\hat{\phi}_{g}(p), g \in \mathcal{U}_{p}\right\}$ is three-dimensional. Suppose, first, that for all $p \in \partial \Sigma$ we have $\left\{\hat{\phi}_{g}(p), g \in\right.$ $\left.\mathcal{U}_{p}\right\}=\{p\}$. It follows that for every Killing vector $\hat{X}$ we have $\hat{X}(p)=0$ for $p \in \partial \mathcal{D}(\Sigma)$. From [35] or 21] it follows that $\partial \mathcal{D}(\Sigma)$ is a Lipschitz continuous surface. By a theorem of Rademacher [14] it follows that $\partial \mathcal{D}(\Sigma)$ has a tangent plane almost everywhere. Now it is not too difficult to adapt the arguments of the proof of Lemma 2.1.1 of [5] to cover the case of such hypersurfaces, to conclude that $\hat{X} \equiv 0$ on $M$ for all Killing vectors, which is obviously not the case.

We have thus shown that $\hat{\phi}_{g}(p)$ cannot act trivially on $\partial \mathcal{D}(\Sigma)$, hence there exist $p \in \partial \mathcal{D}(\Sigma)$ such that $\hat{\phi}_{g}(p) \neq p$ for some $g \in \mathcal{U}_{p}$. Let $p$ be such a point and define

$$
\hat{S}_{p}=\left\{\hat{X} \in \mathcal{V}_{p}: \hat{\phi}_{\exp (\hat{X})}(p)=p\right\} .
$$

Let $S_{p}$ be the linear subspace of $\mathbf{g}$ spanned by $\hat{S}_{p}$ and set

$$
G_{p}=\exp \left(S_{p}\right)
$$

$G_{p}$ is a Lie subgroup of $G$ which does not coincide with $G$, and from the structure of $G$ (recall that $G=S U(2)$ or $S O(3)$ ) it follows that either $G_{p}=\{e\}$, where $e$ is the identity element of $G$, or $G_{p} \approx U(1)$. In the former case the Killing vectors span a three-dimensional subspace of $T_{p} M$, and point 2 follows by setting $\mathcal{U}=\mathcal{U}_{p}, \mathcal{O}=\mathcal{O}_{p}$, and passing to subsets thereof if necessary. Consider then the case $G_{p} \approx U(1)$, let $\hat{X}$ be a generator of $G_{p}$, consider the space $(\hat{X})^{\perp_{k}} \subset \mathbf{g}$, where " $\perp_{k}$ " denotes the orthogonal complement with respect to the Killing metric $k$ on $G$. From the structure of $G$ it follows that for $g \in G_{p}$ the maps $a d_{g}$ act on $(\hat{X})^{\perp_{k}}$ by rotations. This implies that for $0 \neq \hat{Y} \in(\hat{X})^{\perp_{k}}$, the vectors $\hat{Y}(p)$ at $p$ are non-vanishing and spacelike. Let

$$
A_{p}=\left\{X \in T_{p} M: g(X, \hat{Y})=0 \text { for all } \hat{Y} \in(\hat{X})^{\perp_{k}}\right\} .
$$

As the maps $\left(\hat{\phi}_{g}\right)_{p}^{*}$, where $\psi_{p}^{*}$ denotes the pull-back map at $\mathrm{p}$ derived from the map $\psi$, preserve the metric tensor at $p$, it follows that $A_{p}$ is a twodimensional timelike plane invariant under $\left(\hat{\phi}_{g}\right)_{p}^{*}, g \in G_{p}$. Consequently, either $G_{p}$ acts trivially on $A_{p}$, or it acts on it by boosts. In the latter case

${ }^{7}$ In $[5]$ it was assumed that the hypersurface in question is not null. This restriction is, however, not needed for the argument to go through. 
we can find a nearby point $p^{\prime}$ on the null generator of $\partial \mathcal{D}(\Sigma)$ through $p$ such that the Killing vectors span a three-dimensional subspace of $T_{p^{\prime}} M$, and setting $\mathcal{U}=\mathcal{U}_{p^{\prime}}$, and perhaps passing to subsets thereof if necessary point 2 follows. The former case is impossible, which can be seen as follows: if $\left(\hat{\phi}_{g}\right)_{p}^{*}$ acts trivially on $A_{p}$, let $X_{p} \in A_{p}$ be any null vector at $p$ which is not tangent to the generator of $\partial \mathcal{D}(\Sigma)$, let $\hat{\Gamma}$ be a maximally extended null geodesic through $p$ which is tangent to $X_{p}$, set $\Gamma=\hat{\Gamma} \cap \stackrel{\circ}{\mathcal{D}}(\Sigma)$. If $\Gamma$ were not empty, it would be a null geodesic in $\stackrel{\circ}{\mathcal{D}}(\Sigma)$ invariant under $G_{p^{\prime}}$ but there are no null geodesics in $\stackrel{\circ}{\mathcal{D}}(\Sigma)$ invariant under any non-trivial element of $G$.

We have thus shown that there exists $p \in \partial \mathcal{D}(\Sigma)$ such that the Killing vectors span a three-dimensional plane in $T_{p} M$. Indeed, the argument above shows that the set of such $p$ 's is open and dense in $\partial \mathcal{D}(\Sigma)$. Because $\partial \mathcal{D}(\Sigma)$ is invariant under the (local) action $\hat{\phi}_{g}$ for $g \in \mathcal{U}_{p}=\mathcal{U}$, it follows that there exists a neighbourhood $\mathcal{O}=\mathcal{O}_{p} \subset \partial \mathcal{D}(\Sigma)$ of $p$ such that $\partial \mathcal{D}(\Sigma)$ is a submanifold of $M$ of differentability class $C^{k, 1}$, with the action of $\mathcal{U}$ on $\mathcal{O}$ being simply transitive.

When $\partial \mathcal{D}(\Sigma)$ is compact, the following simpler proof of Proposition 2.1 can be given. Arguing as above, the Killing vector fields can be extended by continuity to vector fields on $\overline{\mathcal{D}(\Sigma)}$, tangent to $\partial \mathcal{D}(\Sigma)$. By compactness of $\partial \mathcal{D}(\Sigma)$ every vector field there is complete, so that an action of $G$ is defined on $\partial \mathcal{D}(\Sigma)$. By transitivity of the action of $G$ on Cauchy surfaces, and by the fact that a sequence of Cauchy surfaces accumulating at $\partial \mathcal{D}(\Sigma)$ exists, it follows that the action is transitive on $\partial \mathcal{D}(\Sigma)$. From the list of actions of $G$ given in [15] it follows that the action is free, and by the arguments given above $\partial \mathcal{D}(\Sigma)$ is a differentiable submanifold of differentiability class $C^{k, 1}$.

The orbits of the group action on $\stackrel{\circ}{\mathcal{D}}(\Sigma)$ form a smooth foliation. Denote the future-pointing unit normal to this foliation by $u$. In general $u$ will not have a continuous extension to $\partial \mathcal{D}(\Sigma)$ but the direction it determines does have a $C^{k-1,1}$ extension. Let $\hat{u}$ denote a future-pointing vector field which is proportional to $u$ on $\stackrel{\circ}{\mathcal{D}}(\Sigma)$ and which extends in a $C^{k-1,1}$ manner to $\partial \mathcal{D}(\Sigma)$. (For instance $\hat{u}$ could be taken to be a vector proportional to $u$ which is unit with respect to a smooth auxiliary Riemannian metric.) Now a frame will be constructed which is convenient for calculations. A null frame is by definition either a set of (real) vector fields $(l, n, x, y)$ with $g(l, n)=1, g(x, x)=g(y, y)=-1 / 2$ and all other inner products zero, or the set of vector fields $(l, n, m \equiv x+i y)$ derived from the former. Here and elsewhere $i \equiv \sqrt{-1}$. In the following $l$ and $n$ will be assumed future pointing and Cauchy horizons will be assumed without loss of generality to be future horizons. Thus $l$ and $n$ point from the globally hyperbolic region towards the Cauchy horizon; alternatively we can say that $l$ points away from $\stackrel{\circ}{\mathcal{D}}(\Sigma)$.

Lemma 2.2 Let $\gamma$ be a null geodesic in $\stackrel{\circ}{\mathcal{D}}(\Sigma)$ which has an endpoint $p$ on $\partial \mathcal{D}(\Sigma)$. Then there exist an open neighborhood $\mathcal{V}_{0}$ of $p$ in $\partial \mathcal{D}(\Sigma)$, an open neighborhood $\mathcal{V}$ of $\gamma$ in $\stackrel{\circ}{\mathcal{D}}(\Sigma)$ such that $\overline{\mathcal{V}} \ni \mathcal{V}_{0}$, a neighborhood $\mathcal{U}$ of e in $G$ 
and a smooth null frame $(l, n, x, y)$ on $\mathcal{V}$ such that

1. $l$ is tangent to $\gamma$,

2. the vectors $x$ and $y$ are tangent to the group orbits,

3. the frame is invariant in the sense that if $p, q \in \mathcal{V}, g \in \mathcal{U}$ and $\phi_{g}(p)=q$ then the derivative of $\phi_{g}$ maps the frame at $p$ to the frame at $q$,

4. the Newman-Penrose (NP) coefficients $\epsilon$ and $\kappa$ vanish,

5. the frame has a $C^{k-1,1}$ extension to $\mathcal{V}_{0}$.

Proof: Let $p$ be the endpoint of $\gamma$ on $\partial \mathcal{D}(\Sigma)$ and $l$ a future-pointing tangent vector to $\gamma$ there. There exists a neighbourhood $\mathcal{V}_{0}$ of $p$ in $\partial \mathcal{D}(\Sigma)$ and a neighbourhood $\mathcal{U}$ of $e$ in $G$ such that the mapping $g \mapsto \hat{\phi}_{g}(p)$ of Proposition 2.1 is a $C^{k-1,1}$ diffeomorphism of $\mathcal{U}$ onto $\mathcal{V}_{0}$. Extend $l$ to $\mathcal{V}_{0}$ by invariance under $G$, i.e. if $q \in \mathcal{V}_{0}$ let $l(q)=\left(\hat{\phi}_{g}\right)_{*}(l(p))$, where $g$ is the unique element of $\mathcal{U}$ with $\hat{\phi}_{g}(p)=q$. Here $\psi_{*}$ denotes the tangent map of the map $\psi$. Let $\mathcal{V}$ be the union of the inextendible null geodesics in $\stackrel{\circ}{\mathcal{D}}(\Sigma)$ through points of $\mathcal{V}_{0}$ with initial vector $l$. Extend $l$ to $\mathcal{V}$ by requiring it to be the tangent vector to those geodesics at every point. Now $l$ and $u$ define a smooth $2-$ dimensional distribution $\Pi$ in $\mathcal{V}$. Let $n$ be the unique future-pointing null vector contained in $\Pi$ and satisfying $g(l, n)=1$. Let $x$ and $y$ be smooth vector fields of length $1 / \sqrt{2}$ on $\mathcal{V}$ which are invariant under $G$ and form an orthogonal basis of the orthogonal complement of $\Pi$. The vector field $n$ clearly has a $C^{k-1,1}$ extension to $\partial \mathcal{D}(\Sigma)$ and $x$ and $y$ can be chosen so that they also extend. In this way a frame is obtained which has properties 103 and 5. There remains some arbitrariness in the choice of $x$ and $y$ and this will now be used to arrange $\$$. The fact that $l$ is tangent to a congruence of affinely parametrized null geodesics implies that $\kappa$ vanishes and that $\epsilon$ is purely imaginary.

Let $t$ be an affine parameter on $\gamma$. Then the residual freedom in $x$ and $y$ is a $t$-dependent rotation. Call the angle of rotation $\theta(t)$. Then under a change of basis $\Im \epsilon$, where $\Im \epsilon$ is the imaginary part of $\epsilon$, changes by a constant multiple of $d \theta / d t$. Hence a rotation of $x$ and $y$ can be chosen so that for the new frame $\epsilon=0$.

The vector $u$ is a linear combination of $l$ and $n$. The normalization conditions which have been imposed imply that it is of the form $\frac{1}{\sqrt{2}}\left(z^{1 / 2} l+z^{-1 / 2} n\right)$ for some non-vanishing $\operatorname{smooth}^{\nabla}$ function $z$ on $\stackrel{\circ}{\mathcal{D}}(\Sigma)$. As $\partial \mathcal{D}(\Sigma)$ is approached $u$ becomes parallel to $n$ and hence $z \rightarrow 0$. The NP equations which will be needed will now be written out. As usual in the NP formalism $l$ and $n$ will be denoted by $D$ and $\Delta$ when they are thought of as differential operators on functions. The NP coefficients are invariant under the group and so only

\footnotetext{
${ }^{8} z$ can be seen to be smooth by general considerations. Alternatively its smoothness follows from Proposition 2.4 below.
} 
depend on $t$, the affine parameter along $\gamma$. The same is true of $z$. Hence the differential operators associated to $x$ and $y$ annihilate these quantities and when acting on these quantities $D$ and $\Delta$ are related by $\Delta=z D$. The relevant NP equations can be found in [32, Appendix A] or [42, Appendix $\mathrm{A}]$, we reproduce them here for the convenience of the reader.

$$
\begin{aligned}
D \rho & =\rho^{2}+\sigma \bar{\sigma} \\
D \sigma & =(\rho+\bar{\rho}) \sigma+\Psi_{0} \\
D \tau & =(\tau+\bar{\pi}) \rho+(\bar{\tau}+\pi) \sigma+\Psi_{1} \\
D \alpha & =\rho \alpha+\beta \bar{\sigma}+\rho \pi \\
D \beta & =(\alpha+\pi) \sigma+\bar{\rho} \beta+\Psi_{1} \\
D \gamma & =(\tau+\bar{\pi}) \alpha+(\bar{\tau}+\pi) \beta+\tau \pi+\Psi_{2} \\
D \lambda & =(\rho \lambda+\bar{\sigma} \mu)+\pi^{2}+(\alpha-\bar{\beta}) \pi \\
D \mu & =(\bar{\rho} \mu+\sigma \lambda)+\pi \bar{\pi}-\pi(\bar{\alpha}-\beta)+\Psi_{2} \\
D \nu-z D \pi & =(\pi+\bar{\tau}) \mu+(\bar{\pi}+\tau) \lambda+(\gamma-\bar{\gamma}) \pi+\Psi_{3} \\
z D \Psi_{0} & =(4 \gamma-\mu) \Psi_{0}-2(2 \tau+\beta) \Psi_{1}+3 \sigma \Psi_{2} \\
z D \lambda & =-(\mu+\bar{\mu}) \lambda-(3 \gamma-\bar{\gamma}) \lambda+(3 \alpha+\bar{\beta}+\pi-\bar{\tau}) \nu-\Psi_{4}( \\
0 & =\rho(\bar{\alpha}+\beta)-\sigma(3 \alpha-\bar{\beta})+(\rho-\bar{\rho}) \tau-\Psi_{1} \\
0 & =(\mu \rho-\lambda \sigma)+\alpha \bar{\alpha}+\beta \bar{\beta}-2 \alpha \beta+\gamma(\rho-\bar{\rho})-\Psi_{2} \\
0 & =(\rho-\bar{\rho}) \nu+(\mu-\bar{\mu}) \pi+\mu(\alpha+\bar{\beta})+\lambda(\bar{\alpha}-3 \beta)-\Psi_{3} \\
-z D \mu & =\left(\mu{ }^{2}+\lambda \bar{\lambda}\right)+(\gamma+\bar{\gamma}) \mu-\nu \pi+(\tau-3 \beta-\bar{\alpha}) \nu \\
-z D \beta & =(\tau-\bar{\alpha}-\beta) \gamma+\mu \tau-\sigma \nu-\beta(\gamma-\bar{\gamma}-\mu)+\alpha \bar{\lambda} \\
-z D \sigma & =(\mu \sigma+\bar{\lambda} \rho)+(\tau+\beta-\bar{\alpha}) \tau-(3 \gamma-\bar{\gamma}) \sigma \\
z D \rho & =-(\rho \bar{\mu}+\sigma \lambda)+(\bar{\beta}-\alpha-\bar{\tau}) \tau+(\gamma+\bar{\gamma}) \rho-\Psi_{2} \\
z D \alpha & =\rho \nu-(\tau+\beta) \lambda+(\bar{\gamma}-\bar{\mu}) \alpha+(\bar{\beta}-\bar{\tau}) \gamma-\Psi_{3}
\end{aligned}
$$

These equations hold on $\stackrel{\circ}{\mathcal{D}}(\Sigma)$ and all the quantities occuring there are bounded in a (one-sided) neighborhood of $\partial \mathcal{D}(\Sigma)$. Using the equations it can be seen that all quantities except possibly $\Psi_{0}$ extend continuously to $\partial \mathcal{D}(\Sigma)$.

A central part of this paper is to discuss the possible solutions of the above set of equations and the first step is to discuss the solution on $\partial \mathcal{D}(\Sigma)$. By applying the commutator equations which define the NP coefficients to an arbitrary $G$-invariant function Siklos 42 obtains the equations

$$
\begin{aligned}
D z & =-(\gamma+\bar{\gamma}), \\
0 & =\kappa z+(\bar{\alpha}+\beta-\bar{\pi}), \\
0 & =-\bar{\nu}+(\tau-\bar{\alpha}-\beta) z, \\
0 & =\mu-\bar{\mu}+(\rho-\bar{\rho}) z .
\end{aligned}
$$

Thus on $\partial D(\Sigma)$ we must have $\pi=\alpha+\bar{\beta}, \nu=0$ and $\Im \mu=0$. He also shows, by transforming from the null frame to a more conventional orthonormal 
frame, that Bianchi class A solutions (which include those of type IX) satisfy

$$
\begin{aligned}
\mu+\rho z & =0, \\
\tau-2 \beta-\kappa z & =0 .
\end{aligned}
$$

Hence in this case $\mu=0$ and $\tau=2 \beta$ on the horizon. The mean extrinsic curvature of the orbits is given by $\sqrt{2} \Re\left\{(\epsilon-\bar{\rho}) z^{1 / 2}+(\mu-\bar{\gamma}) z^{-1 / 2}\right\}$, where $\Re(x)$ or $\Re x$ denotes the real part of $x$, which for a class A solution is equal to $-\sqrt{2} \Re \gamma z^{-1 / 2}+O\left(z^{1 / 2}\right)$. In [37] it was shown that for Bianchi IX spacetimes the mean extrinsic curvature must tend monotonically to infinity at the boundary of the maximal Cauchy development ( $c f$. Lemma 4.2 below for a detailed argument) and so it can be concluded that $\gamma+\bar{\gamma} \neq 0$ on $\partial \mathcal{D}(\Sigma)$.

Lemma 2.3 Let $\gamma$ be a null geodesic as in Lemma 2.9. Then there exists a possibly different null geodesic $\bar{\gamma}$ with the same endpoint such that on $\partial \mathcal{D}(\Sigma)$ the only non-vanishing NP coefficients of the frame associated to $\bar{\gamma}$ as in Lemma 2.8 are $\gamma$ and $\rho$. Moreover $\bar{\gamma} \rho+\gamma \bar{\rho}=0$ there.

Proof: From the above discussion we know that $\nu=0, \mu=0, \tau=2 \beta$ and $\gamma+\bar{\gamma} \neq 0$ on $\partial \mathcal{D}(\Sigma)$. A null rotation about $n$ can be used to set $\sigma$ to zero 42]. The geodesic $\bar{\gamma}$ is chosen to have the transformed $l$ as tangent vector. The remaining statements follow from the NP equations (2.12)-(2.24) at $z=0$ by straightforward algebra.

Proposition 2.4 Consider the system of equations (2.9)-(2.11) and (2.21) for the vector valued function

$$
f=\left(\rho, \sigma, \tau, \alpha, \beta, \gamma, \lambda, \mu, \nu, z, \Psi_{0}\right) \in \mathbf{C}^{11},
$$

in which the $\Psi_{a}$ 's, $a=1,2,3$ are determined by $f$ via eqs (2.15) 2.15), and $\pi$ is determined by (2.29) with $\kappa=0$. Suppose moreover that $\gamma$ and $\mu$ are continuous and that

$$
\Re \gamma(0) \neq 0, \quad \Re\left(\frac{4 \gamma-\mu}{\gamma+\bar{\gamma}}\right)(0)>0 .
$$

Assume finally that $z$ is continuous with $z(0)=0$, and $z(x)>0$ for $x \neq 0$. Then:

1. Let $\epsilon>0$ and let $f \in L^{\infty}\left([0, \epsilon] ; \mathbf{C}^{11}\right) \cap C^{1}\left((0, \epsilon) ; \mathbf{C}^{11}\right)$ satisfy 甲 these equations. Then $f$ can be extended by continuity to a continuous function on $[0, \epsilon]$, still denoted by $f$, such that

$$
f \in C^{\infty}\left([0, \epsilon] ; \mathbf{C}^{11}\right) .
$$

\footnotetext{
${ }^{9}$ For an open set $\Omega$ we use the standard notation $C^{1}(\Omega ; X)$ to denote the collection of $X$-valued functions on $\Omega$ which are differentiable on $\Omega-$ no uniform bounds are implied; similarly for spaces with higher derivative index. On the other hand $C^{k}(\bar{\Omega} ; X)$, respectively $C^{\infty}(\bar{\Omega} ; X)$ denote the spaces of those smooth functions on $\Omega$ the derivatives of which of order less than or equal to $k$, respectively of all orders, can be extended by continuity to continuous functions on the closure $\bar{\Omega}$ of $\Omega . L^{\infty}(\Omega ; X)$ denotes the collection of Lebesgue-measurable $X$-valued functions on $\Omega$ which are essentially bounded on $\Omega$.
} 
2. Let $\epsilon>0$ and let $f_{1}, f_{2} \in C^{0}\left([0, \epsilon] ; \mathbf{C}^{11}\right) \cap C^{1}\left((0, \epsilon), \mathbf{C}^{11}\right)$ be two solutions of those equations satisfying our previous hypotheses, with

$$
f_{1}(0)=f_{2}(0)
$$

Then

$$
f_{1} \equiv f_{2}
$$

Proof: Writing $f$ as $\left(g, \Psi_{0}\right)$ and using coordinates in which $D=-\frac{d}{d x}$, equations (2.2)-2.11) together with eq. (2.21) are of the form

$$
x \frac{d}{d x}\left(\begin{array}{c}
g \\
\Psi_{0}
\end{array}\right)=\left(\begin{array}{c}
x B(f) \\
C(f, x)
\end{array}\right),
$$

where

$$
C(f, x)=\frac{x}{z} E(f),
$$

with $E(f)$ given by the right hand side of eq. (2.11), and $B(f)$ given by the right hand side of eqs. (2.2) $-(2.10)$ and (2.21). Note that by the first part of (2.25) and by (2.21) we can choose $\delta$ small enough so that $x / z$ is bounded on $[0, \delta]$. From our hypotheses on $f$ it follows immediately that $d g / d x \in L^{\infty}\left([0, \epsilon] ; \mathbf{C}^{10}\right)$, so that $g$ can be uniquely extended to a Lipschitz continuous function on $[0, \epsilon]$, still denoted by $g$. From (2.21) we then have $\lim _{x \rightarrow 0} x / z=1 /(2 \Re \gamma)(0)$. The equation for $\Psi_{0}$ can be rewritten in the form

$$
x \frac{d}{d x}\left(\Psi_{0}(x)-\Psi_{0}(0)\right)+\chi(x)\left(\Psi_{0}(x)-\Psi_{0}(0)\right)=F(x),
$$

with

$$
\chi(x)=-\frac{(4 \gamma-\mu) x}{z}, \quad \Re \chi(0)=\Re\left\{\frac{4 \gamma-\mu}{2 \Re \gamma}\right\}>0 .
$$

Here the constant $\Psi_{0}(0)$ has been chosen so that $F(0)=0$; that this can always be achieved follows from our hypotheses which guarantee that $\chi(0) \neq$ 0 . Integrating (2.28) it follows that there exists a constant $C$ such that

$$
\Psi_{0}(x)-\Psi_{0}(0)=\left[C-\int_{x}^{\epsilon} \frac{F(s)}{s} \exp \left(-\int_{s}^{\epsilon} \frac{\chi(t)}{t} d t\right) d s\right] \exp \left(\int_{x}^{\epsilon} \frac{\chi(t)}{t} d t\right) .
$$

Clearly $\left|\Psi_{0}\right|$ will blow up as $x^{-\Re \chi(0)}$ at $x=0$ unless the constant $C$ is appropriately chosen. Let us set

$$
\Xi(x)=\exp \left(\int_{0}^{x} \frac{\chi(0)-\chi(s)}{s} d s\right) .
$$

From (2.29) and from our hypothesis $\Psi_{0} \in L^{\infty}([0, \epsilon], \mathbf{C})$ it therefore follows

$$
\Psi_{0}(x)-\Psi_{0}(0)=\left[\int_{0}^{x} \frac{F(s)}{\Xi(s)} s^{\chi(0)-1} d s\right] x^{-\chi(0)} \Xi(x),
$$

which immediately gives

$$
\left|\Psi_{0}(x)-\Psi_{0}(0)\right| \leq C x
$$


An easy bootstrap of (2.30) and of (2.26) establishes point 1. To prove point 2 , let $f_{a}=\left(g_{a}, \Psi_{0}^{a}\right), a=1,2$, be two solutions and define

$$
\begin{gathered}
\Delta g(x)=g_{1}(x)-g_{2}(x), \\
\Delta \Psi(x)=\Psi_{0}^{1}(x)-\Psi_{0}^{2}(x), \\
H=x^{-1}\langle\Delta g, \overline{\Delta g}\rangle+x^{-1 / 2}|\Delta \Psi|^{2} .
\end{gathered}
$$

Here a bar denotes complex conjugation, and $\langle$,$\rangle is the standard scalar$ product on $\mathbf{R}^{10}$. We have

$$
\frac{d H}{d x}=-x^{-2}\langle\Delta g, \overline{\Delta g}\rangle-\frac{1}{2} x^{-3 / 2}|\Delta \Psi|^{2}+G,
$$

where by $G$ we have denoted the sum of all the remaining terms which arise by differentiation of $H$. One of those terms, e.g., will be

$$
x^{-3 / 2}\left\{\Delta \bar{\Psi}\left[\frac{x}{z_{1}} E\left(f_{1}\right)-\frac{x}{z_{2}} E\left(f_{2}\right)\right]+c . c .\right\},
$$

with $E(f)$ as in (2.27), and where c.c. denotes the complex conjugate term. This can be rewritten as

$$
x^{-3 / 2}\left\{\Delta \bar{\Psi}\left[\frac{x \Delta z}{z_{1} z_{2}} E\left(f_{1}\right)+\frac{x}{z_{2}}\left(E\left(f_{1}\right)-E\left(f_{2}\right)\right)\right]+\text { c.c. }\right\} .
$$

In what follows the letter $C$ will denote a large constant which may vary from expression to expression. Now by eq. (2.21) for $x$ small enough we have $z_{a} \geq C^{-1} x, a=1,2$. Moreover (2.26) and $\Psi_{0}^{1} \in C^{1}([0, \epsilon] ; \mathbf{C})$ imply that $\left|E\left(f_{1}\right)\right| \leq C x$. Consequently, for any $\alpha>0$ we have

$$
\begin{aligned}
x^{-3 / 2}\left|\Delta \bar{\Psi} \frac{x \Delta z}{z_{1} z_{2}} E\left(f_{1}\right)\right| & \leq C x^{-3 / 2}|\Delta \Psi||\Delta z| \\
& \leq \frac{C}{\alpha} x^{-3 / 2}|\Delta z|^{2}+\frac{C \alpha}{4} x^{-3 / 2}|\Delta \Psi|^{2} .
\end{aligned}
$$

The second term in the right-hand-side of this equation will be dominated by the second term in (2.31) if we choose $\alpha$ small enough. Then we can find $\epsilon_{1}>0$ such that for $x \in\left(0, \epsilon_{1}\right)$ the first term in $(2.33)$ will be dominated by the first term in (2.31).

To analyze the remaining terms in (2.32), let us write the expression $E(f)$ in eq. (2.27) in the form

$$
E(f)=-\chi(g)\left(\Psi_{0}-\Psi_{0}(0)\right)+F(g) .
$$

We then have

$$
\begin{gathered}
\Delta \bar{\Psi}\left(E\left(f_{1}\right)-E\left(f_{2}\right)\right)+c . c .= \\
=\Delta \bar{\Psi}\left(-\chi\left(g_{1}\right)\left(\Psi_{0}^{1}-\Psi_{0}(0)\right)+\chi\left(g_{2}\right)\left(\Psi_{0}^{2}-\Psi_{0}(0)\right)+F\left(g_{1}\right)-F\left(g_{2}\right)\right)+c . c . \\
=\left\{-\chi\left(g_{1}\right)|\Delta \Psi|^{2}+\Delta \bar{\Psi}\left[\left(\chi\left(g_{2}\right)-\chi\left(g_{1}\right)\right)\left(\Psi_{0}^{2}-\Psi_{0}(0)\right)+F\left(g_{1}\right)-F\left(g_{2}\right)\right]\right\}+c . c . \\
\left.=-2 \Re \chi\left(g_{1}\right)|\Delta \Psi|^{2}+\left\{\Delta \bar{\Psi}\left(\chi\left(g_{2}\right)-\chi\left(g_{1}\right)\right) \Psi_{0}^{2}+F\left(g_{1}\right)-F\left(g_{2}\right)\right)+c . c .\right\} .
\end{gathered}
$$


The first term in this expression is negative for $x$ small enough, and can be dropped, while the remaining terms can be estimated as in the previous calculation. Proceeding similarly with the remaining terms which occur in $G$ in (2.31) we find that there exists $0<\delta \leq \epsilon$ such that for $x \in(0, \delta)$ we have

$$
\frac{d H}{d x} \leq 0
$$

As $H$ is continuous on $[0, \delta]$ with $H(0)=0$ we obtain

$$
H \equiv 0 \Rightarrow f^{1} \equiv f^{2}
$$

on $[0, \delta] . f^{1} \equiv f^{2}$ on $[0, \epsilon]$ follows now from standard uniqueness results for (non-singular) ODE's.

It has now been shown that in a Bianchi IX spacetime with a Cauchy horizon the spin coefficients in a certain frame take a special form on the horizon. It has also been shown that the spin coefficients are determined everywhere by their values on the horizon. The spin coefficients of certain frames in the Taub-NUT spacetimes will be calculated and compared with the restrictions previously obtained. The Taub-NUT metrics can be written (perhaps locally) in the form [30

$$
\begin{gathered}
U^{-1} d t^{2}-\left[(2 L)^{2} U \sigma_{1}^{2}+\left(t^{2}+L^{2}\right)\left(\sigma_{2}^{2}+\sigma_{3}^{2}\right)\right], \\
U(t)=-1+\frac{2\left(m t+L^{2}\right)}{t^{2}+L^{2}} .
\end{gathered}
$$

where $\sigma_{1}, \sigma_{2}$ and $\sigma_{3}$ are, say, left invariant one-forms on $S U(2)$. The constants $L$ and $m$ are real numbers with $L>0$. Let $X_{1}, X_{2}, X_{3}$ be dual basis vectors to $\sigma_{1}, \sigma_{2}$ and $\sigma_{3}$. They have the commutation relations $\left[X_{i}, X_{j}\right]=\epsilon^{k}{ }_{i j} X_{k}$. Two null vectors can be defined by

$$
\begin{aligned}
l & =\frac{1}{\sqrt{2}}\left(\frac{\partial}{\partial t} \pm \frac{1}{2 L U} X_{1}\right), \\
n & =\frac{1}{\sqrt{2}}\left(U \frac{\partial}{\partial t} \mp \frac{1}{2 L} X_{1}\right) .
\end{aligned}
$$

They can be completed to a basis by $x=\frac{1}{\sqrt{2\left(t^{2}+L^{2}\right)}} X_{2}$ and $y=\frac{1}{\sqrt{2\left(t^{2}+L^{2}\right)}} X_{3}$. The non-vanishing spin coefficients of the frame $(l, n, x, y)$ are given by

$$
\begin{gathered}
\rho=\frac{1}{\sqrt{2}}\left(-\frac{t}{t^{2}+L^{2}} \pm \frac{L}{t^{2}+L^{2}} i\right), \\
\mu=\frac{1}{\sqrt{2}}\left(\frac{U t}{t^{2}+L^{2}} \mp \frac{L U}{t^{2}+L^{2}} i\right), \\
\epsilon=\frac{1}{2 \sqrt{2}}\left( \pm \frac{L}{t^{2}+L^{2}} \mp \frac{1}{2 L U} i\right), \\
\gamma=\frac{1}{2 \sqrt{2}}\left[-\dot{U}+\left(\mp \frac{L U}{t^{2}+L^{2}} \pm \frac{1}{2 L}\right) i\right] .
\end{gathered}
$$

Here and elsewhere a dot over a quantity denotes a time derivative. Since $\epsilon \neq 0$ this is not a frame of the type used by Siklos and constructed in Lemma 2.2. However, as in the proof of that lemma, $\epsilon$ can be set to zero by 
doing a rotation of $x$ and $y$. This rotation leaves the other spin coefficients except $\gamma$ unchanged. In the new frame

$$
\gamma=\frac{1}{2 \sqrt{2}}\left[-\dot{U}+\left(\mp \frac{2 L U}{t^{2}+L^{2}} \pm \frac{1}{L}\right) i\right] .
$$

On the future horizon $\dot{U}=-\frac{4 t\left(m^{2}+L^{2}\right)}{\left(t^{2}+L^{2}\right)^{2}}$ and it can be checked directly that $\bar{\gamma} \rho+\gamma \bar{\rho}=0$ there.

In Lemma 2.3 it was shown that on the Cauchy horizon of a Bianchi IX space-time $\Re(\gamma \bar{\rho})=0$. The restriction $\gamma+\bar{\gamma} \neq 0$ was also obtained. The fact that a future horizon is considered means that in fact $\gamma+\bar{\gamma}>0$. There is a further restriction which follows from the criteria given $\square$ by Siklos [42, Section 3], namely that $(\Im \gamma)(\Im \rho)<0$. Define

$$
S_{1}=\left\{(\rho, \gamma) \in \mathbf{C}^{2}: \Re(\gamma \bar{\rho})=0, \gamma+\bar{\gamma}>0,(\Im \gamma)(\Im \rho)<0\right\} .
$$

The family of spin coefficients arising from Taub-NUT space-times described above can be extended by using a constant boost of $l$ and $n$. Under a boost $\rho$ and $\gamma$ transform according to $\rho \rightarrow A \rho, \gamma \rightarrow A^{-1} \gamma$, where $A$ is an arbitrary positive real number. A calculation reveals that the resulting family of spin coefficients depending on the three parameters $L, m$ and $A$ precisely exhausts the set $S_{1}$. (The two components of $S_{1}$ defined by the sign of $\Im \gamma$ correspond to the two possible signs in the definition (2.36)-(2.37) of $l$ and $n$.) Thus given a Bianchi IX space-time with a horizon there exists a Taub-NUT space-time and a null frame there which has the same spin coefficients as a frame in the given space-time.

Lemma 2.5 Let $(M, g)$ be a vacuum Bianchi IX space-time with partial Cauchy surface $\Sigma$, as defined at the beginning of this section and let $p$ be a point of $\partial \mathcal{D}(\Sigma)$. Then there exists a Taub-NUT space-time $\left(M^{\prime}, g^{\prime}\right)$ and an isometry $\phi_{p}$ of a neighbourhood $\mathcal{W}_{p}$ in $\overline{\mathcal{D}(\Sigma)}$ of $p$ onto a subset of $\left(M^{\prime}, g^{\prime}\right)$.

Proof: It has already been shown that there exist a null geodesic $\gamma$ with endpoint $p$, a Taub-NUT space-time $\left(M^{\prime}, g^{\prime}\right)$ and a null geodesic $\gamma^{\prime}$ in $M^{\prime}$ with endpoint $p^{\prime}$ such that the frames associated to $\gamma$ and $\gamma^{\prime}$ as in Lemma 2.2 have the same spin coefficients. If $q$ belongs to the neighbourhood $\mathcal{V}_{0}$ of $p$, let $g$ be the unique element of $\mathcal{U}$ with $\hat{\phi}_{g}(p)=q$ and define $\phi(q)=\phi_{g}^{\prime}\left(p^{\prime}\right)$, where $\phi^{\prime}$ denotes the action of $G=S U(2)$ or $G=S O(3)$ on the Taub-NUT space-time. The mapping $\phi$ is a diffeomorphism of $\mathcal{V}_{0}$ onto a neighbourhood of $p^{\prime}$ in the horizon of the Taub-NUT solution. The frame on the TaubNUT space-time is not uniquely defined by $\gamma$ and $\gamma^{\prime}$ alone. There remains

\footnotetext{
${ }^{10}$ Let us elaborate slightly on this point. By choice the function $z$ has been chosen to be positive on $\stackrel{\circ}{\mathcal{D}}(\Sigma)$. Now if we choose $l$ to be pointing away from $\stackrel{\circ}{\mathcal{D}}(\Sigma)$, then 2.21 implies that $\Re \gamma$ must be nonnegative on the Cauchy horizon. Alternatively we could ask that $l$ be future pointing - in that case we have $\Re \gamma \geq 0$ on a future Cauchy horizon and $\Re \gamma \leq 0$ on a past one.

${ }^{11}$ The reader should be warned of misprints in eq. (3.2) of 42 .
} 
the freedom to do Lorentz transformations which preserve $n$. However the requirement that the NP coefficients be identical fixes the two null vectors in each case uniquely. Thus $\phi_{*, p}(l)=l^{\prime}$ and $\phi_{*, p}(n)=n^{\prime}$. Here $\phi_{*, p}$ denotes the tangent map at $p$ associated to the map $\phi$. The vectors $\phi_{*, p}(x)$ and $\phi_{*, p}(y)$ may differ from $x^{\prime}$ and $y^{\prime}$ by a rotation. However this rotation does not change the NP coefficients and so we may define $x^{\prime}=\phi_{*, p}(x)$ and $y^{\prime}=\phi_{*, p}(y)$. Now the frame at $p^{\prime}$ agrees with the image under $\phi$ of the frame at $p$. Using the group invariance shows that the frames agree on $\partial \mathcal{D}(\Sigma)$. The mapping $\phi$ will now be extended to a neighbourhood of $p$. Let $q$ be a point which lies on a null geodesic with endpoint on $\mathcal{V}_{0}$ and has tangent vector $l$ there. Let $r$ be the endpoint of this geodesic and $t$ the affine distance from $r$ to $q$. Define $\phi(q)$ to be the point obtained by following the geodesic through $\phi(r)$ with tangent vector $l^{\prime}$ there an affine distance $t$ into the past. To complete the proof of the lemma it suffices to show that $\phi$ is an isometry and to do that it is enough to show that $\phi$ maps the frame $(l, n, x, y)$ to the frame $\left(l^{\prime}, n^{\prime}, x^{\prime}, y^{\prime}\right)$ everywhere (note that at this stage we know only that this is true on a subset of the horizon). To see that this is true, note first that the construction of $\phi$ ensures that $\phi_{*} l=l^{\prime}$ wherever defined. The definition of the NP coefficients allows the Lie derivatives of $n$, $x$ and $y$ to be expressed as linear combinations of $l, n, x$ and $y$, where the coefficients are expressions involving the NP coefficients. Of course the same is true of the Lie derivatives of $n^{\prime}, x^{\prime}$ and $y^{\prime}$. Because the spin coefficients are the same for both frames this means that the components of $\phi_{*} n, \phi_{*} x, \phi_{*} y$ and those of $n^{\prime}, x^{\prime}, y^{\prime}$ satisfy the same system of linear ordinary differential equations. Since $\phi_{*} n=n^{\prime}, \phi_{*} x=x^{\prime}$ and $\phi_{*} y=y^{\prime}$ on $\partial \mathcal{D}(\Sigma)$ they also have the same initial data at some point. Hence $\phi_{*} n=n^{\prime}, \phi_{*} x=x^{\prime}$ and $\phi_{*} y=y^{\prime}$ on a neighbourhood of $p$.

Proof of Theorem 1.1: It follows from the construction of the local isometry $\phi$ in the proof of Lemma 2.5 that $\phi$ maps the intersection of each hypersurface of homogeneity with $\mathcal{W}_{p}$ into a hypersurface of constant $t$ in the Taub-NUT spacetime. Here $t$ is as in eq. (2.34). Let $(\gamma, K)$ be the initial data which $(M, g)$ induces on the hypersurface of homogeneity $\Sigma_{q}$ containing a point $q \in \mathcal{W}_{p} \cap \mathcal{D}(\Sigma)$ and let $\left(\gamma^{\prime}, K^{\prime}\right)$ be the initial data on the corresponding hypersurface $\Sigma_{q}^{\prime}$ of constant $t$ in Taub-NUT spacetime. Then $\phi_{*} \gamma=\gamma^{\prime}$ and $\phi_{*} K=K^{\prime}$, wherever the left hand sides of these equations are defined. Now if $\Sigma_{q}^{\prime}$ is simply connected, it can be identified naturally with $S U(2)$, and then $\gamma^{\prime}, K^{\prime}$ are left-invariant tensors there. It can be assumed without loss of generality that $\phi(q)$ is identified with $e$. If $\Sigma_{q}$ is simply connected then there is a diffeomorphism $\phi_{1}: \Sigma_{q} \rightarrow S U(2)$ with $\phi_{1}(q)=e$ such that $\left(\phi_{1}\right)_{*} \gamma$ and $\left(\phi_{1}\right)_{*} K$ are left-invariant. Now $\phi_{1} \circ \phi$ is a mapping $S U(2) \rightarrow S U(2)$ such that $\left(\phi_{1} \circ \phi\right)_{*} \gamma^{\prime}=\phi_{1 *} \gamma$ on a neighbourhood of the identity. Since both of these tensors are left invariant they must be equal everywhere. A similar argument applies to $K$. Hence $\phi$ maps $\left(\Sigma_{q}, \gamma, K\right)$ onto $\left(\Sigma_{q}^{\prime}, \gamma^{\prime}, K^{\prime}\right)$ and their maximal globally hyperbolic developments must be isometric. It follows that $\mathcal{D}(\Sigma)$ is isometric to (the globally hyperbolic part of) a Taub-NUT spacetime. If $\Sigma_{q}$ is not simply connected we get the 
statement that $\mathcal{D}(\Sigma)$ is isometric to a quotient of a Taub-NUT spacetime by a discrete group of isometries, which are the Taub-NUT spacetimes with lens space spatial topology.

To finish the proof of Theorem 1.1 we need to prove that generic Bianchi IX initial data on $L(p, 1), p=1,2$, are not of Taub-NUT type. The result is well known on $L(1,1)=S^{3}$, cf. e.g. [5] or [39]. For $p>1$ recall that $L(p, 1)$ is defined as $S^{3} / \mathbf{Z}_{p}$, for a suitable action of $\mathbf{Z}_{p}$ on $S^{3}$. We have the following:

Proposition 2.6 1. All Bianchi IX initial data on $S^{3}$ pass to the quotient $S^{3} / \mathbf{Z}_{2} \approx L(2,1)$.

2. For $p>2$ all (globally) homogeneous Bianchi IX initial data on $L(p, 1)$ are of Taub-NUT type.

Remark: Let us emphasize that there exist non-Taub-NUT Bianchi IX metrics on $L(p, 1)$ for any $p>1$; $c f$. the discussion in Section 3 below. These will, however, be only locally homogeneous for $p>2$ if they are not of Taub-NUT type.

Proof: Using the Euler angle parametrization of $S^{3}$ (cf. e.g. eq. (5.1) below) the relevant $\mathbf{Z}_{p}$ action consists of translations in $\mu$ and $\varphi$ :

$$
\mu \rightarrow \mu+\frac{2 \pi}{p}, \quad \varphi \rightarrow \varphi+\frac{2 \pi}{p} .
$$

Let $\phi$ denote the corresponding map from $S^{3}$ into itself. Consider ${ }^{2}$ the Killing vectors $X_{a}, a=1,2,3$ given by equations (17a)-(17c) of [8]. Let $\sigma^{b}$, $b=1,2,3$, be the corresponding dual forms, $\sigma^{a}\left(X_{b}\right)=\delta_{b}^{a}$. From (2.39) one immediately obtains

$$
\phi^{*}\left[\begin{array}{l}
\sigma^{1} \\
\sigma^{2} \\
\sigma^{3}
\end{array}\right]=\left[\begin{array}{ccc}
\cos \frac{2 \pi}{p} & -\sin \frac{2 \pi}{p} & 0 \\
\sin \frac{2 \pi}{p} & \cos \frac{2 \pi}{p} & 0 \\
0 & 0 & 1
\end{array}\right]\left[\begin{array}{l}
\sigma^{1} \\
\sigma^{2} \\
\sigma^{3}
\end{array}\right] .
$$

Any $S U(2)$ invariant two-covariant symmetric tensor $f$ on $S^{3}$ can be written in the form

$$
f=f_{i j} \sigma^{i} \otimes \sigma^{j}
$$

where $f_{i j}$ is a symmetric 3 by 3 constant coefficients matrix. It easily follows from (2.40) that 1) if $p=2$, then $f$ passes to the quotient if and only if $\left.f_{13}=f_{23}=0,2\right)$ if $p>2$, then $f$ passes to the quotient if and only if $f$ is diagonal with $f_{11}=f_{22}$. Our claims follow directly from the above.

\footnotetext{
${ }^{12}$ To obtain the standard form of the $S U(2)$ commutation relations the vectors $X_{1}$ and $X_{2}$ given in [8] should be interchanged.
} 


\section{Some remarks on local vs. global degrees of free- dom}

The issue of strong cosmic censorship for spatially locally homogeneous spacetimes of a given Bianchi type cannot in general be decided by an analysis of the universal covering spacetimes alone. If Cauchy horizons could be ruled out for all the covering spacetimes of a given Bianchi type then there would be nothing left to be done. However the typical situation is that Cauchy horizons can only be ruled out for almost all of the covering spacetimes and that there are exceptional cases where a Cauchy horizon does exist. A given choice of the topology of a compactification may be incompatible with almost all covering spacetimes but compatible with the exceptional geometries. This will now be illustrated by the example of vacuum Bianchi I spacetimes. In that case the three-metric on the initial surface in the covering spacetime may be assumed without loss of generality to be the standard flat metric on $\mathbf{R}^{3}$. Compactifications are then obtained by factoring out by discrete subgroups of the Euclidean group which leave the second fundamental form invariant. In $\mathbf{R}^{3}$ the tangent space at any point can be naturally identified with the tangent space at the origin. This identification leads to a homomorphism from the Euclidean group to the rotation group $O(3)$. By homogeneity the second fundamental form is constant when its values at different points are compared using the natural identification. Hence the question of which geometries can be compactified reduces to the following question. Given a discrete subgroup $H$ of the Euclidean group which defines a compactification, let $\bar{H}$ be the corresponding subgroup of $O(3)$. Which constant symmetric tensors $K$ on $\mathbf{R}^{3}$ are invariant under $\bar{H}$ ? It must also be remembered that only a $K$ which satisfies the Hamiltonian constraint is of interest in the present context.

There are three cases to be considered, according to whether the number of distinct eigenvalues of $K$ is one, two or three. In the first case the Hamiltonian constraint implies that $K=0$. Any group $H$ is allowed. This is flat space-time identified in a way which is compatible with a timelike Killing vector. The resulting maximal globally hyperbolic developments of the initial data are static and geodesically complete. In the second case the Hamiltonian constraint implies that the eigenvalues of $K$ must be of the form $(\lambda, 0,0)$ or $\left(\frac{2}{3} \lambda, \frac{2}{3} \lambda,-\frac{1}{3} \lambda\right)$. These correspond to the $(1,0,0)$ and $\left(\frac{2}{3}, \frac{2}{3},-\frac{1}{3}\right)$ Kasner solutions respectively. The $(1,0,0)$ Kasner solutions admit a Cauchy horizon and in fact these are the only choices of $K$ compatible with a Cauchy horizon. In particular the third case is not compatible with a Cauchy horizon. The possible groups $H$ which lead to a compact orientable quotient manifold have been listed by Koike, Tanimoto and Hosoya in [27], and we shall consider their list one by one. The first type on their list is the torus, where the group $\bar{H}$ is trivial. There all three cases are possible for $K$ and cosmic censorship holds in the sense that there is no Cauchy horizon for a generic metric with Bianchi I symmetry. The second type, where the only non-trival element of $\bar{H}$ is a rotation through $\pi$ is similar. In the next 
three types $\bar{H}$ consists of rotations about a given axis including ones through angles other than $\pi$. Hence only the first and second cases are possible for $K$. Thus there are two classes of solutions without Cauchy horizons and one with Cauchy horizons. Since the class with Cauchy horizons is not in any obvious sense smaller than the set of solutions without horizons, it seems reasonable to say that cosmic censorship fails in this case. Finally, in the sixth type $\bar{H}$ contains rotations about distinct axes so that only the first case is possible for $K$. For this topology all Bianchi I spacetimes are geodesically complete and cosmic censorship holds.

It should be emphasized that the various cases discussed above arise because the isometries used to identify the universal covering space did not form a subgroup of the Bianchi group considered. Indeed, consider a simply connected Bianchi group and let $\Gamma$ be any discrete subgroup thereof. Let $g$ be any left-invariant metric on $G$, then $g$ passes to the manifold obtained by dividing $G$ by the left action of $\Gamma$ on $G$. The resulting metric will, however, only be locally homogeneous in general. This follows from the fact that the Killing vector fields of the resulting metric on the group manifold are generated by left translations of $G$ on itself, which are not globally defined on the coset space in general. This is e.g. what happens in the case of Bianchi IX's on higher $p$ lens spaces; here the $\mathbf{Z}_{p}$ action comes from an action of $G$ on itself so that any metric passes to an appropriate quotient to yield a locally homogeneous metric.

To close this section, let us point out that even in the case of a solution with a Cauchy horizon and a group which can be used to make identifications in the globally hyperbolic region, the Cauchy horizon may not survive the identifications. Consider the chronological future of the origin in Minkowski spacetime. The group corresponding to Bianchi type $\mathrm{V}$ can be made to act on this region so that the orbits are the hyperboloids of constant Lorentzian distance from the origin. In this way this part of Minkowski spacetime can be regarded as a Bianchi V spacetime. This region can be factored out by the action of a discrete subgroup of the Lorentz group so as to become spatially compact. However, it follows from Proposition 4.7 below that the resulting spacetime cannot be extended through a Cauchy horizon.

\section{Spatially locally homogeneous space-times with Cauchy horizons}

In this section we shall show how to generalize the results of Section 2 to the spatially locally homogeneous case. Let $(\Sigma, \gamma, K)$ be a vacuum initial data set, let $\hat{\Sigma}$ be the universal cover of $\Sigma$ and let $\hat{\gamma}, \hat{K}$ be the lifts of $\gamma$ and $K$ to $\hat{\Sigma}$. We shall say that $(\Sigma, \gamma, K)$ are locally homogeneous if there exists a group $G$ acting transitively on $\hat{\Sigma}$ such that its action leaves $\hat{\gamma}$ and $\hat{K}$ invariant. We could similarly define a Riemannian manifold $(\Sigma, \gamma)$ to be locally homogeneous by dropping $K$ in our definition above. This 
apparently differs from the standard definition given e.g. in [43], where local homogeneity is defined by postulating existence of an appropriate set of locally defined isometries. When $\Sigma$ is complete a theorem of Singer [43] shows that these two definitions are actually equivalent. The approach using locally defined isometries seems to be somewhat more natural, as it does not require the introduction of universal covers, etc. We have chosen the above definition of local homogeneity of initial data since no analogue of Singer's theorem including $K$ is known. The definition which makes use of the universal cover turns out to be very convenient.

Let us start with a brief discussion of the Kantowski-Sachs symmetry type. These models correspond to the $S^{2} \times \mathbf{R}$ model discussed in Section 2.4.8 of [27. The corresponding initial data surfaces are obtained by taking compact quotients of $S^{2} \times \mathbf{R}$, with the corresponding initial data on $S^{2} \times \mathbf{R}$ being invariant under the obvious action of $S O(3) \times \mathbf{R}$ on $S^{2} \times \mathbf{R}$. It is well known and in any case easily seen that the latter initial data can be obtained as data induced on a cylinder $r=$ const $<2 m$ in an extended Schwarzschild space-time. Similarly the maximal globally hyperbolic development of such data can be obtained by taking quotients of the $r<2 m$ region in the extended Schwarzschild space-time. It follows that there is always a curvature singularity in one time direction $(r \rightarrow 0)$. Moreover when the initial data surface is compact, then the space-time can always be extended across a compact Cauchy horizon in the other time direction, $r \rightarrow 2 m$. This shows that SCC "half-fails" in this class of space-times. Note, however, that the local algebra of Killing vectors is always four-dimensional, which establishes the Kantowski-Sachs part of Theorem 1.4.

To analyze the Bianchi symmetry type, we shall need the following result (it should be emphasized that compactness of $\Sigma$ is not assumed here):

Theorem 4.1 Let $(\Sigma, \gamma, K)$ be locally homogeneous vacuum initial data, with $(\Sigma, \gamma)$ - geodesically complete, and let $(M, g)$ be the maximal globally hyperbolic development thereof. Then there exists on $M$ a time function $t \in$ $C^{\infty}(M)$ with range $(-\infty, 0)$ the level sets of which are locally homogeneous Cauchy surfaces.

Remark: Theorem 4.1 allows us to define the notion of hypersurfaces of homogeneity, as the level sets of the function $t$ given there.

Proof: Let $\hat{\Sigma}$ be the universal cover of $\Sigma$ with projection map $\pi$, set $\hat{\gamma}=$ $\pi^{*} \gamma, \hat{K}=\pi^{*} K$, let $G$ be the group appearing in the definition of local homogeneity, let $(\hat{M}, \hat{g})$ be the maximal globally hyperbolic development of $(\hat{\Sigma}, \hat{\gamma}, \hat{K})$. Recall also that $\pi_{1}(\Sigma)$ acts on $\hat{\Sigma}$, and for $g \in \pi_{1}(\Sigma)$ the action $\pi_{g}$ thereof satisfies $\pi \circ \pi_{g}=\pi$. We have

$$
\pi_{g}^{*} \hat{\gamma}=\pi_{g}^{*} \pi^{*} \gamma=\left(\pi \circ \pi_{g}\right)^{*} \gamma=\hat{\gamma},
$$

similarly for $g \in \pi_{1}(\Sigma)$ one has $\pi_{g}^{*} \hat{K}=\hat{K}$. By Theorem 2.1.4 of [5] there exist actions $\hat{\phi}_{g}$ of $G$ and $\hat{\pi}_{g}$ of $\pi_{1}(\Sigma)$ on $(\hat{M}, \hat{g})$ by isometries such that $\hat{\phi}_{g}\left|\hat{\Sigma}=\phi_{g}, \hat{\pi}_{g}\right| \hat{\Sigma}=\pi_{g}$. 
Let $p \in \hat{\Sigma}$ and let $\hat{\Gamma}=\{p(s)\}_{s \in I}$ be a maximally extended affinely parametrized timelike geodesic normal to $\hat{\Sigma}$ such that $p(0)=p$. Here $I$ is some open connected subset of $\mathbf{R}$. Define a family of surfaces $\hat{\Sigma}_{s}, s \in I$ by

$$
\hat{\Sigma}_{s}=\left\{\phi_{g}(p(s)), g \in G\right\} .
$$

It is clearly seen that the $\hat{\Sigma}_{s}$ 's cover $\hat{M}$ and that the function defined as $t_{1}(p)=s$ for $p \in \hat{\Sigma}_{s}$ is a smooth time function on $\hat{M}$. Let $f: I \rightarrow(-\infty, 0)$ be any diffeomorphism, set $\hat{t}=f \circ t_{1}$. The function $\hat{t}$ can be used to induce a diffeomorphism

$$
\hat{M} \approx \hat{\Sigma} \times(-\infty, 0)
$$

in a standard way. Define now

$$
M=\hat{M} / \pi_{1}(\Sigma) .
$$

Note that for $g \in \pi_{1}(\Sigma)$ we have $\hat{t} \circ \pi_{g}=\hat{t}$ so that $\hat{t}$ passes to the quotient and defines a time-function $t \in C^{\infty}(M)$. In particular we have

$$
M \approx \Sigma \times(-\infty, 0) .
$$

We wish to show that $M$ is globally hyperbolic. Indeed, $M$ is stably causal since it possesses a time function ( $c f .$, e.g., 445). Moreover $\Sigma \approx \Sigma \times\left\{t_{0}\right\} \subset M$ is acausal as it is a level set of $t$. Let $\Gamma$ be a causal curve in $M$, let $p \in \Gamma$, choose $\hat{p} \in \pi^{-1}(\Gamma)$ and let $\hat{\Gamma}$ be the connected component of $\pi^{-1}(\Gamma)$ through $\hat{p}$. By global hyperbolicity of $\hat{M}$ the curve $\hat{\Gamma}$ can be extended if necessary to a curve $\hat{\Gamma}^{\prime}$ such that $\hat{\Gamma}^{\prime} \cap\{\hat{\Sigma}\} \neq \emptyset$, setting $\Gamma^{\prime}=\pi\left(\hat{\Gamma}^{\prime}\right)$ one obtains an extension of $\Gamma$ which intersects $\Sigma$, and global hyperbolicity follows.

It remains to show that $M$ is maximal globally hyperbolic. While one can give a general argument using properties of maximal globally hyperbolic developments and universal covers, we have found it useful to present the alternative proof that follows, as the arguments below give some information about the global structure of $(M, g)$.

The following result has been essentially proved in [37:

Lemma 4.2 Let $(M, g)$ be a vacuum spacetime which is the maximal globally hyperbolic development of (locally) homogeneous initial data. Let $\tau$ be a coordinate which is constant on the hypersurfaces of homogeneity and which coincides with a proper time parameter when restricted to geodesics orthogonal to these hypersurfaces. Denote the mean extrinsic curvature of the hypersurface labelled by $\tau$ by $H(\tau)$. Then $H$ is monotone and:

1. For Bianchi IX or Kantowski-Sachs symmetry typeß in each time direction $|H|$ tends to infinity in finite time.

2. For the remaining symmetry types, $H$ runs from $-\infty$ to 0 (changing time orientation if necessary). The spacetime exists globally in $\tau$ in 
that time direction in which $|H|$ tends to zero, say $\tau \in\left(-\infty, \tau_{+}\right)$, for some $\mathrm{I}^{[3} \tau_{+} \in \mathbf{R} \cup \infty$, and $\lim _{\tau \rightarrow-\infty} H=0$.

Proof: To see this it suffices to note the following facts. Firstly, the results in [37] are stated for the Vlasov-Einstein system, where the matter content of spacetime is described by a distribution function $f$, but the vacuum case is included as the case where $f$ is identically zero. Secondly, since the arguments used there are local in space, the distinction between homogeneity and local homogeneity is irrelevant. All the results of the lemma for a spacetime with Bianchi symmetry except the last (that $|H|$ tends to zero) follow from Lemma 2.2 of [37] and the discussion immediately following it. The corresponding results for a spacetime with Kantowski-Sachs symmetry follow from Lemma 3.1 of [37], or can be derived by direct calculations in the Schwarzschild space-time. It also follows that if $H$ remains bounded for $\tau$ greater than some $\tau_{0}$ then $H<0$ for $\tau>\tau_{0}$. The inequality $\partial_{\tau} H \geq \frac{1}{3} H^{2}$ ((2.35) in [37]) then implies that $H \rightarrow 0$ as $\tau \rightarrow \infty$. A similar argument applies if $\tau$ is replaced by $-\tau$ and this completes the proof of the lemma.

Returning to the proof of Theorem 4.1, by Lemma 4.2 the mean extrinsic curvature $H(s)$ of the slices $\Sigma_{s}=\{p \in M \mid t(p)=s\}$ tends in modulus either to infinity or to zero as $s$ approaches 0 or $-\infty$. The inextendability of $(M, g)$ in the class of globally hyperbolic space-times in that time direction in which $H$ blows up follows now from Hawking's focussing Lemma 21, Prop. 4.4.3] (cf., e.g., [5, Prop. C.2.5]). It follows from [36, Theorem 2.1] that the spacetime must be causally geodesically complete in that time direction in which $H$ tends to zero (if any), from which inextendability again follows.

To finish the proof of Theorem 4.1, we need to show that all level sets of $t$ are Cauchy surfaces. In the case of compact $\Sigma$ this follows immediately from [2] (cf. also [18]). In the general case the arguments above show that this will follow if we show that the level sets of $\hat{t}$ are Cauchy surfaces. Consider then a surface $\hat{\Sigma}_{t_{0}}$, by [0], Prop. 2.4] $\stackrel{\circ}{\mathcal{D}}\left(\hat{\Sigma}_{t_{0}} ; \hat{M}\right)$ is the maximal globally hyperbolic development of $\left(\Sigma_{t_{0}}, \hat{\gamma}_{t_{0}}, \hat{K}_{t_{0}}\right)$, where $\left(\hat{\gamma}_{t_{0}}, \hat{K}_{t_{0}}\right)$ are the initial data induced on $\hat{\Sigma}_{t_{0}}$ from $\hat{g}$. By [5, Theorem 2.1.4] G acts on $\stackrel{\circ}{\mathcal{D}}\left(\hat{\Sigma}_{t_{0}} ; \hat{M}\right)$ by isometries. It is easily seen that $G$ acts transitively on all the $\hat{\Sigma}_{t}$ 's, so that we must have $\partial \stackrel{\circ}{\mathcal{D}}\left(\hat{\Sigma}_{t_{0}} ; \hat{M}\right) \cap \hat{\Sigma}_{t}=\emptyset$ for all $t \in(-\infty, 0)$. Hence $\partial \stackrel{\circ}{\mathcal{D}}\left(\hat{\Sigma}_{t_{0}} ; \hat{M}\right)=\emptyset$, which completes the proof.

Consider now $p \in \Sigma$, let $\hat{p} \in \pi^{-1}(p)$ and let $\hat{\mathcal{O}}$ be a neighbourhood of $\hat{p}$ such that $\pi: \hat{\mathcal{O}} \leftrightarrow \mathcal{O}$ is a diffeomorphism. For any Killing vector field $\hat{X} \in \mathbf{g}$ there exists a corresponding Killing vector field $X=\pi_{*} X$ defined on $\mathcal{O}$. For $g \in G$ let $\hat{\phi}_{g}$ denote the action of $G$ on $\hat{M}$, for $\epsilon>0$ set

$$
\mathcal{O}(p, \epsilon)=\pi\left(\left\{\hat{q}=\phi_{\exp (\hat{X})}(\hat{p}):|\hat{X}|<\epsilon\right\}\right) .
$$

There exists $\epsilon(p)>0$ such that for all $0<\epsilon \leq \epsilon(p)$ the sets $\mathcal{O}(p, \epsilon)$ are connected, simply connected open neighbourhoods of $p$. We have the following:

\footnotetext{
${ }^{13}$ The case $\tau_{+}=\infty$ can occur only if $(M, g)$ is obtained by some identifications from Minkowski space-time.
} 
Lemma 4.3 Under the hypotheses of Theorem 4.1, suppose moreover that $\Sigma$ is compact. Let $\Sigma_{t}$ be the level sets of the function $t$ given by Theorem 4.1. For every $\epsilon>0$ there exists $I(\epsilon)<\infty$ such that for any set of points $\left\{p_{i}\right\}_{i=1}^{I} \subset \Sigma_{t}$ there exist $i, j$ such that

$$
\mathcal{O}\left(p_{i}, \epsilon\right) \cap \mathcal{O}\left(p_{j}, \epsilon\right) \neq \emptyset .
$$

Remark: Let us emphasize that $I(\epsilon)$ given here is $t$-independent.

Proof: Let $\stackrel{\circ}{\mathcal{B}}(p, s)$ denote the open geodesic ball of radius $s$ centered at $p$, where the distance is measured with respect to the metric $\gamma$ on $\Sigma \approx \Sigma_{t}$. (Here we use the diffeomorphism $M \approx(-\infty, 0) \times \Sigma$ to transport the metric $\gamma$ from $\Sigma$ to $\left.\Sigma_{t}\right)$. Choose $q_{0} \in \Sigma$ and let $\epsilon_{1}(\epsilon)$ be such that $\stackrel{\circ}{\mathcal{B}}\left(q_{0}, \epsilon_{1}\right)$ is diffeomorphic to an open ball in $\mathbf{R}^{3}$, and that $\stackrel{\circ}{\mathcal{B}}\left(q_{0}, \epsilon_{1}\right) \subset \mathcal{O}\left(q_{0}, \epsilon\right)$. By $G$-invariance of $\gamma$ it then follows that for all $p \in \Sigma$ we shall also have $\stackrel{\circ}{\mathcal{B}}\left(p, \epsilon_{1}\right) \subset \mathcal{O}(p, \epsilon)$. From the covering $\left\{\stackrel{\circ}{\mathcal{B}}\left(q, \epsilon_{1} / 3\right)\right\}_{q \in \Sigma}$ of $\Sigma$ a finite covering $\left\{\stackrel{\circ}{\mathcal{B}}\left(q_{i}, \epsilon_{1} / 3\right)\right\}_{i=1}^{J}$ can be chosen.

Let $I(\epsilon)$ be the largest integer larger than or equal to $J / 2$. To see that $I(\epsilon)$ satisfies our assertions consider any set of points $\left\{p_{i}\right\}_{i=1}^{I}$. Because the balls $\stackrel{\circ}{\mathcal{B}}\left(q_{i}, \epsilon_{1} / 3\right)$ cover $\Sigma$, every point $p \in \Sigma$ is a distance at least $\epsilon_{1} / 3$ for some $q_{i(p)}$. Note, moreover, that every $\mathcal{O}\left(p_{i}, \epsilon\right)$ contains a ball of radius $\epsilon_{1}$, and therefore at least two points $q_{i}$. It follows that any collection of more than $\lceil J / 2\rceil$ sets $\mathcal{O}\left(p_{i}, \epsilon\right)$ has to have at least one intersection $\mathcal{O}\left(p_{i}, \epsilon\right) \cap \mathcal{O}\left(p_{j}, \epsilon\right)$ non-empty.

Let $G$ be the connected component of the identity of the group of isometries of the space-times $(\hat{M}, \hat{g})$ discussed in the proof of Theorem 4.1, and let $\mathbf{g}$ be the Lie algebra of $G$. We have the following:

Proposition 4.4 Let $(\Sigma, \gamma, K)$ be locally homogeneous vacuum initial data on a compact manifold $\Sigma$, consider a space-time $(M, g)$ which is a (nontrivial) extension of the maximal globally hyperbolic development of $(\Sigma, \gamma, K)$, with a metric of differentiability class $C^{k, 1}, k \geq 1$. Then for every $p \in \partial \mathcal{D}(\Sigma)$ there exists an open neighbourhood $\mathcal{O}_{p} \subset M$ of $p$ such that

1. On $\mathcal{O}_{p} \cap \stackrel{\circ}{\mathcal{D}}(\Sigma)$ there exists a Lie algebra $A_{p}$ of Killing vectors isomorphic to $\mathbf{g}$.

2. The vector fields $X \in A_{p}$ extend $C^{k, 1}$ continuously to $\partial \mathcal{D}(\Sigma)$.

3. The orbits of $X \in A_{p}$ through $p \in \partial \mathcal{D}(\Sigma)$ are three-dimensional.

Remark: Point 3 above does not hold if the compactness condition is relaxed. As an example, consider the unit spacelike hyperboloid in Minkowski space-time with the corresponding induced Cauchy data. The maximal globally hyperbolic development of the initial data is isometrically diffeomorphic to the interior of the light cone of the origin. Taking the Minkowski 
space-time as the extension, the Cauchy horizon is then the light-cone of the origin, with the orbits of the symmetry group through the origin being 0-dimensional. In this example, however, there exists an open dense subset of the horizon on which the orbits are three-dimensional. We believe that this is always true, even if the compactness of $\Sigma$ is not assumed.

Proof: Let $\mathcal{O}_{p} \subset M$ be any neighbourhood of $p \in \partial \mathcal{D}(\Sigma)$ such that $\mathcal{O}_{p} \cap \stackrel{\circ}{\mathcal{D}}(\Sigma)$ is connected and simply connected. Choose some point $q \in \mathcal{O}_{p} \cap \stackrel{\circ}{\mathcal{D}}(\Sigma)$. As explained in the discussion preceeding Lemma 4.3, there exists a neighbourhood of $q$ and a Lie algebra of Killing vector fields isomorphic to $\mathbf{g}$ defined there. A theorem of Hall [20] ( $c f$. also [33, 43]) shows that the Killing vectors can be extended to $\mathcal{O}_{p} \cap \stackrel{\circ}{\mathcal{D}}(\Sigma)$, and point 1 follows. Point 2 follows as in the proof of Proposition 2.1, Section 2 .

To establish point 3 , let $K=\left\{p \in \partial \mathcal{D}(\Sigma) \mid\right.$ the orbits of $x \in A_{p}$ through $p$ are not three-dimensional $\}$. Consider a point $q \in K$, there exists a neighbourhood $\mathcal{U}_{q} \subset \mathcal{O}_{q} \cap \partial \mathcal{D}(\Sigma)$ of $q$ and $\epsilon>0$ such that $\phi_{\exp (X)}(p)$ is defined for all $p \in \mathcal{U}_{q}$ and $|X|<\epsilon$ by following the integral curve of $X$ through $p$ a parameter-distance one. Clearly $\partial \mathcal{D}(\Sigma) \backslash K$ is open, we want to show that it is the whole of $\partial \mathcal{D}(\Sigma)$. In order to do this consider $p \in \partial \mathcal{D}(\Sigma)$ such that the orbit

$$
\mathcal{O}(p, 5 \epsilon)=\left\{\phi_{\exp (X)}(p), \quad|X|<5 \epsilon\right\}
$$

is not three-dimensional. Reducing $\epsilon$ if necessary $\mathcal{O}(p, 5 \epsilon)$ is a smooth embedded submanifold of $M$; further reducing $\epsilon$ if required we can find a neighbourhood $\mathcal{O}$ of $p$ such that $\phi_{\exp (X)}(q)$ is defined for all $q \in \mathcal{O}$ and for all $|X| \leq 4 \epsilon$. We claim that there exists a sequence $p_{i} \in \mathcal{O}, i \in \mathbf{N}$, such that

$$
\mathcal{O}\left(p_{i}, \epsilon\right) \cap \mathcal{O}\left(p_{j}, \epsilon\right)=\emptyset \quad \text { if } \quad i \neq j .
$$

We shall proceed by induction. Thus, let $p_{1}$ be any point in $\mathcal{O} \backslash \mathcal{O}(p, 5 \epsilon)$. Suppose that a sequence $p_{i} \in \mathcal{O} \backslash \mathcal{O}(p, 5 \epsilon), i=1, \ldots I$, satisfying (4.1) has been constructed for some $I \geq 1$. Set

$$
\Omega=\cup_{i=1}^{I} \overline{\mathcal{O}\left(p_{i}, 2 \epsilon\right)}
$$

We have $\Omega \cap \overline{\mathcal{O}(p, 2 \epsilon)}=\emptyset$, which implies that there exists a neighbourhood $\mathcal{O}_{I} \subset \mathcal{O}$ of $p$ such that $\Omega \cap \mathcal{O}_{I}=\emptyset$. As $\mathcal{O}\left(p_{1}, 5 \epsilon\right)$ is a smooth embedded submanifold of dimension less than three, there exists a point $p_{I+1} \in$ $\mathcal{O}_{I} \backslash \mathcal{O}(p, 5 \epsilon)$. As $p_{I+1} \notin \Omega$, one has for all $i=1, \ldots I$

$$
\mathcal{O}\left(p_{i}, \epsilon\right) \cap \mathcal{O}\left(p_{I+1}, \epsilon\right)=\emptyset
$$

so that the induction step is completed. [Clearly the sequence $\left\{p_{i}\right\}$ can be so chosen so that $p_{i} \rightarrow p$, this will however not be relevant in what follows.]

Let $T$ be any timelike vector field defined on $\mathcal{U}_{q}$, we define $q_{i}(s)$ as the intersection of the orbit of $T$ through $q_{i}$ with the level sets $\Sigma_{s}$ of the function $t \in C^{\infty}(\stackrel{\circ}{\mathcal{D}}(\Sigma))$ given by Theorem 4.1. Here $t$ is chosen so that $t(p) \rightarrow 0$ as $p \rightarrow \partial \mathcal{D}(\Sigma)$. Let $I(\epsilon)$ be given by Lemma 4.3 , choose any set $\left\{q_{i}\right\}_{i=1}^{I(\epsilon)}$. 
Now the sets $\left\{\phi_{\exp (X)}\left(q_{i}\right)|| X \mid<\epsilon\right\}$ are mutually disjoint, and continuous dependence of solutions of ODE's upon initial values shows that for $|s|$ small enough the sets $\left\{\phi_{\exp (X)}(q(s))|| X \mid<\epsilon\right\}$ will be mutually disjoint, which contradicts Lemma 4.3 and proves the result.

Proof of Theorem 1.4: Suppose now that a spatially locally homogeneous space-time with a compact partial Cauchy surface is of Bianchi class A. Then the obvious analogues of Lemma 2.2 and Lemma 2.3 hold. There exists a null frame for which the only non-vanishing NP coefficients on the horizon are $\rho$ and $\gamma$. Moreover $\Re(\gamma \bar{\rho})=0$ and $\Re \gamma>0$. The region $S_{1}$, where $(\Im \gamma)(\Im \rho)<0$ was discussed in the previous section, $c f$. eq. (2.38). Consider now the following subsets of $\mathbf{C}^{2}$ :

$$
\begin{gathered}
S_{2}=\left\{(\rho, \gamma) \in \mathbf{C}^{2}: \Re(\gamma \bar{\rho})=0, \Re \gamma>0,(\Im \gamma)(\Im \rho)>0\right\}, \\
S_{3}=\left\{(\rho, \gamma) \in \mathbf{C}^{2}: \Re(\gamma \bar{\rho})=0, \Re \gamma>0, \Im \gamma=0, \Im \rho \neq 0\right\}, \\
S_{4}=\left\{(\rho, \gamma) \in \mathbf{C}^{2}: \Re(\gamma \bar{\rho})=0, \Re \gamma>0, \Im \gamma \neq 0, \rho=0\right\}, \\
S_{5}=\left\{(\rho, \gamma) \in \mathbf{C}^{2}: \Re(\gamma \bar{\rho})=0, \Re \gamma>0, \Im \gamma=\Im \rho=0\right\} .
\end{gathered}
$$

Just as a point of $S_{1}$, can only arise from a space-time of type IX, points of $S_{2}, S_{3}, S_{4}$ and $S_{5}$ can only arise from space-times of types VIII, II, VII and I respectively. This can be seen by applying the criterial1 given by Siklos [42, Section 3] for the different Bianchi types in terms of the NP coefficients on the horizon. This identification of the possible Lie algebras is already enough to give an interesting conclusion concerning the Bianchi types of class A mentioned in Proposition 1.3: there are no Cauchy horizons in spatially locally homogeneous vacuum space-times of Bianchi types $\mathrm{VI}_{0}$ admitting a compact partial Cauchy surface. What has been said above shows that this statement remains true without the compactness condition as long as we assume that the Cauchy horizon is locally homogeneous. The vacuum space-times of Bianchi type I are the (possibly twisted) Kasner solutions. It is known that most of these do not possess a Cauchy horizon. The only ones which do are those which are flat ${ }^{\text {t4 }}$. The spin coefficients in the set $S_{4}$ arise from these same space-times; the Bianchi $\mathrm{VII}_{0}$ frame is obtained from the Bianchi I frame by a suitable rotation of $x$ and $y$. More precisely, any locally homogeneous metric of Bianchi I symmetry type can be represented as a locally homogeneous metric of Bianchi $\mathrm{VII}_{0}$ symmetry type by passing from a local basis of Killing vectors $\partial / \partial x, \partial / \partial y$ and $\partial / \partial z$ to the locally defined basis $\partial / \partial x, \partial / \partial y$ and $y \partial / \partial x-x \partial / \partial y+\partial / \partial z$. To complete the picture of which spatially compact class A solutions admit Cauchy horizons it remains to study the type II and type VIII solutions. Space-times which realize the remaining values of the NP coefficients can be obtained by making certain replacements in the Taub-NUT metric. Suppose first that the vectors $X_{1}, X_{2}$ and $X_{3}$ have the commutation relations $\left[X_{1}, X_{2}\right]=-X_{3}$, $\left[X_{2}, X_{3}\right]=X_{1},\left[X_{3}, X_{1}\right]=-X_{2}$ and replace $U$ by $-U$. The NP coefficients of the resulting space-time differ on the horizon from the Taub-NUT case only in that the signs of $\Im \gamma$ and $\Re \rho$ are reversed. They realize the points of

\footnotetext{
${ }^{14}$ Those space-times have been overlooked in the list given in 42, Theorem 2]
} 
$S_{2}$. To obtain the NP coefficients in $S_{3}$ do a similar thing with the commutation relations $\left[X_{1}, X_{2}\right]=0,\left[X_{2}, X_{3}\right]=X_{1},\left[X_{3}, X_{1}\right]=0$ and $U$ replaced by $\tilde{U}=\frac{2 m t}{t^{2}+L^{2}}$. This time the NP coefficients differ from those in the TaubNUT case by the facts that $\Im \gamma=\Re \rho=0$ and that $U$ is replaced by $\tilde{U}$. These are the generalized NUT space-times of types VIII and II studied by Siklos 41]. (They can be compactified, as will be shown below.)

It was shown in [17] that for all spatially compact space-times with Bianchi B symmetry type the algebra of Killing vectors tangent to the surfaces of homogeneity must be at least four dimensional, and Theorem 1.4 follows.

To prove Proposition 1.3 we shall need several auxiliairy results. The following result, which will be used later, has some interest of its own:

Proposition 4.5 Let $(M, g)$ be a vacuum spacetime which is the maximal globally hyperbolic development of locally homogeneous initial data on a compact Cauchy surface $\Sigma$. If $(M, g)$ has an extension $\left(M^{\prime}, g^{\prime}\right)$ through a future Cauchy horizon then it has a possibly different extension $\left(M^{\prime \prime}, g^{\prime \prime}\right)$ through a smooth compact future Cauchy horizon.

Remark: The extension we are constructing here needs not to be vacuum beyond the new Cauchy horizon, even if $\left(M^{\prime}, g^{\prime}\right)$ were.

Proof: As discussed previously all vacuum globally hyperbolic locally homogeneous space-times with Kantowski-Sachs symmetry type are known, and the result is true for those space-times, it is therefore sufficient to consider the Bianchi symmetry type. Let $G$ be the unique simply connected Lie group corresponding to the given Bianchi type. Then there exists a discrete group $\Gamma$ of tranformations of $G$ such that the spacetime $M$ can be identified with $G / \Gamma \times(-\infty, 0)$. Moreover the pullback of $g$ to $G \times(-\infty, 0)$ is invariant under the action of $G$ by left translations. If we denote a point of $G \times(-\infty, 0)$ by $(x, t)$ then $t$ can be chosen such that a future Cauchy horizon in any extension of $(M, g)$ corresponds to $t \rightarrow 0$. Let $\gamma$ be a null geodesic in $\left(M^{\prime}, g^{\prime}\right)$ which crosses the future Cauchy horizon at a point $p$. The identification of $M$ with $G / \Gamma \times(-\infty, 0)$ can be chosen so that the points of $\gamma$ all correspond to the $\Gamma$-orbit of the identity of $G$. It was shown above that the local action of $G$ on $M$ can be extended to a local action of $G$ on the union $\mathcal{V}$ of $M$ with an open neighbourhood of $p$ in the Cauchy horizon. This action can be used to extend the identification of $M$ with $G / \Gamma \times(-\infty, 0)$ to an identification of $\mathcal{V}$ with $\mathcal{W}=G / \Gamma \times(-\infty, 0) \cup \mathcal{U} \times\{0\}$, where $\mathcal{U}$ is an open neighbourhood of the orbit of the identity. Let $\hat{\mathcal{W}}$ be the inverse image of $\mathcal{W}$ under the projection of $G \times(-\infty, 0]$ onto $G / \Gamma \times(-\infty, 0]$. Using the action of the group the pull back to $\hat{\mathcal{W}}$ of the restriction of $g^{\prime}$ to $\mathcal{W}$ can be extended to a $G$-invariant Lorentz metric on $G \times(-\infty, 0]$. It is also $\Gamma$-invariant by continuity since the metric on the region $t<0$ is $\Gamma$-invariant. Taking the quotient by the group $\Gamma$ gives a "space-time with boundary" $G / \Gamma \times(-\infty, 0]$, which is clearly smoothly extendable to some (perhaps nonvacuum) Lorentzian space-time $\left(M^{\prime \prime}, g^{\prime \prime}\right)$ which contains a compact Cauchy horizon. 
We shall also need the following lemma:

Lemma 4.6 Under the hypotheses of Theorem 1.4 suppose that $(M, g)$ is of Bianchi B symmetry type. Then $(M, g)$ is not flat. Moreover the group of isometries of the universal cover of $\mathcal{D}(\Sigma)$ contains a subgroup of Bianchi type III.

Proof: By Lemma 4.5 without loss of generality we may assume that $\partial \mathcal{D}(\Sigma)$ is compact and smooth. It then follows from [21, p. 297] that $\left.\mu\right|_{\partial \mathcal{D}(\Sigma)}=0$. Lemma 4.2 and the discussion before Lemma 2.3 show that $\left.\Re \gamma\right|_{\partial \mathcal{D}(\Sigma)} \neq 0$. Recall now that for any $B \in \mathbf{C}$ the "gauge-transformation" of the tetrad on $\partial \mathcal{D}(\Sigma)$

$$
n^{a} \rightarrow n^{a}, \quad m^{a} \rightarrow m^{a}+\bar{B} n^{a}, \quad l^{a} \rightarrow l^{a}+B m^{a}+\bar{B} m^{a}+B \bar{B} n^{a},
$$

preserves the null-orthonormality conditions. With a little work one finds that under this transformation we have

$$
\sigma \rightarrow \sigma+\bar{B}(\tau+2 \beta)+\bar{B}^{2}(\mu+2 \bar{\gamma})+\bar{B}^{3} \nu .
$$

As $\left.(\mu+2 \bar{\gamma})\right|_{\partial \mathcal{D}(\Sigma)} \neq 0$ we can achieve $\left.\sigma\right|_{\partial \mathcal{D}(\Sigma)}=0$ by performing such a transformation. Hence without loss of generality we may assume that $\left.\sigma\right|_{\partial \mathcal{D}(\Sigma)}=0$. The equations (5.3)-(5.8) of [42] lead then to the following two sets of values of NP coefficients at $\partial \mathcal{D}(\Sigma)$ (in each case below all the unlisted $\mathrm{NP}$ coefficients vanish at $\partial \mathcal{D}(\Sigma))$ :

1. $\rho \in \mathbf{C} \backslash\{0\}, \gamma \in \mathbf{R} \backslash\{0\}, \alpha \in \mathbf{C}$ subject to $|\alpha|^{2}=1 / 3(\bar{\gamma} \rho+\gamma \bar{\rho})$, $\tau=\bar{\alpha}$

2. $\rho \in \mathbf{C} \backslash\{0\}, \gamma \in \mathbf{R} \backslash\{0\}, \alpha \in \mathbf{C}$ subject to $|\alpha|^{2}=1 / 4(\bar{\gamma} \rho+\gamma \bar{\rho})$, $\beta=-\bar{\alpha} ;$

(the case $\rho=0$ leads to a Bianchi class A symmetry type). Note that the hypotheses of Proposition 2.4 are again satisfied. The corresponding metrics are therefore uniquely determined by the values (modulo gauges) of the NP coefficients at $\partial \mathcal{D}(\Sigma)$. It is easily checked that the metrics one obtains in this way are of Bianchi III symmetry type. From eqs. (2.11)-(2.15) one finds that the corresponding metrics are not flat at the Cauchy horizon. (The two families of boundary values of NP coefficients listed above are gauge-equivalent in the sense that they correspond to the same space-time metrics Th. This is, however, irrelevant for our purposes.)

There has been recently some interest in the global structure of maximal globally hyperbolic flat Lorentzian space-times. Let us point out the following:

\footnotetext{
${ }^{15}$ We are grateful to S. Siklos for pointing this out.
} 
Proposition 4.7 Let $(M, g)$ be a spatially compact, locally homogeneous, maximal globally hyperbolic $n+1$ dimensional flat Lorentzian manifold, $n=$ $1,2,3$. If the surfaces of homogeneity are not quotients of an $n$-dimensional torus, then $(M, g)$ is inextendible.

Proof: Multiplying $(M, g)$ by $S^{1}$ when $n=3$ or by $S^{2}$ when $n=2$ and equipping the resulting space-time with the obvious flat product metric we can without loss of generality assume that $n=4$. First the possibility of Kantowski-Sachs symmetry will be eliminated. In that case the universal covering spacetime is spherically symmetric. Define a function $r$ by the condition that its value at any point is such that the area of the orbit through that point under the action of $S O(3)$ is $4 \pi r^{2}$. Define the mass function by $m=(r / 2)\left(1+\nabla_{\alpha} r \nabla^{\alpha} r\right)$. The function $r$ is necessarily constant on the hypersurfaces of homogeneity and so its gradient is timelike. Hence $m$ is positive. However a direct calculation of the curvature of a spherically symmetric spacetime shows that it can only be flat if $m=0$. Hence $(M, g)$ is a flat four dimensional Lorentzian manifold with Bianchi symmetry type. Suppose that $(M, g)$ is extendible. By Proposition 4.5 we can assume that the extension is across a compact Cauchy horizon. By Lemma 4.6 the symmetry type must be Bianchi A, and the result follows from the list of extendible Bianchi A vacuum space-times given in the proof of Theorem 1.4.

Some similar results have been established $\square$ by G. Mess by rather more involved techniques.

Proof of Proposition 1.3: For the Kantowski-Sachs models solutions with compact Cauchy horizons can be obtained by identifying $(t, r, \vec{\omega}) \in$ $\mathbf{R} \times(0,2 m) \times S^{2}$ with $(t+a, r, R \vec{\omega})$, where $a \in \mathbf{R}$ and $R \in S O(3)$ [27], in a Schwarzschild manifold. For the Bianchi I symmetry type the relevant metrics are the flat Kasner metrics, which can be spatially compactified if desired. For the Bianchi $\mathrm{VII}_{0}$ type these are again the flat Kasner metrics, as described in the proof of Theorem 1.4. For the Bianchi II, III $=\mathrm{VI}_{-1}, \mathrm{IV}$, VI - IX symmetry types these are the space-times listed in Theorem 2 of [42]. (Another family of Bianchi III space-times extendible across a Cauchy horizon (both compact and non-compact, if desired) is given by the family of "pseudo-Schwarzschild" solutions (cf. e.g. [13, p. 73, "A2 solutions"] and [16]). The needed Bianchi V space-time which is extendible across a (non-compact) Cauchy horizon can be taken to be the interior of the light cone of the origin in Minkowski space-time. It follows from Proposition 4.7 that spatially compact quotients of this space-time are inextendible.

The NUT spacetimes of Bianchi types VIII and II can both be spatially compactified, as will now be shown. Let $(N, h)$ be a Riemannian manifold, $P$ a principal $U(1)$ bundle over $N$ and $A$ a connection on $P$. There exists a unique Riemannian metric on each fibre invariant under the action of $U(1)$ such that the length of the fibre is $2 \pi$. Call this metric $k$. Given the above data and a positive function $a$ on $M$, a Riemannian metric $\gamma$ on $P$ can be

\footnotetext{
${ }^{16}$ G. Mess, private communication; cf. also 29.
} 
defined as follows. If $X$ and $Y$ are horizontal let $\gamma(X, Y)=h\left(\pi_{*} X, \pi_{*} Y\right)$, where $\pi$ is the projection of $P$ onto $N$. If $X$ is horizontal and $Y$ vertical let $\gamma(X, Y)=0$. If both $X$ and $Y$ are vertical let $\gamma(X, Y)=a^{2} k(X, Y)$. The action of $U(1)$ on $P$ coming from the principal bundle structure is an action by isometries of $\gamma$. As an example of this construction, consider the case where $(N, h)$ is 2-dimensional, $P$ is the unit tangent bundle, $A$ is the Levi-Civita connection and $a=1$. Each isometry of $h$ induces an isometry of $\gamma$ in an obvious way in this example. Moreover, these isometries are all distinct from the $U(1)$ isometry group which all these metrics possess and so the isometry group of $\gamma$ is at least one dimension greater than that of $h$. Applying this construction to the hyperbolic plane gives a metric which by the above has at least a four-dimensional isometry group. Moreover the isometries of $\gamma$ which are induced by orientation-preserving isometries of the base act transitively on $P$. Since the group of orientation-preserving isometries of the hyperbolic plane has a Lie algebra of Bianchi type VIII the metric $\gamma$ is homogeneous with Bianchi VIII symmetry. Now we could have done the same construction starting from a compact quotient of the hyperbolic plane and in that case $\gamma$ is locally homogeneous with Bianchi VIII symmetry and is defined on a compact manifold which is the total space of a circle bundle over a compact surface. It also has an additional global Killing vector given by the $U(1)$ action. In terms of appropriate one-forms on $P$ invariant under the group the metric takes the form $\sigma_{1}^{2}+\sigma_{2}^{2}+\sigma_{3}^{2}$ and $\sigma_{2}$, $\sigma_{3}$ vanish on vectors tangent to the fibres. A smooth family of metrics may be generated from this single one by multiplying $h$ by constant conformal factors and taking general constant values of $a$ in the above construction. This proves that the type VIII NUT metrics are compactifiable in a variety of ways.

Consider next the type II metrics. They can also be compactified with the aid of the above construction but unfortunately not using the unit tangent bundle. (The unit tangent bundle of a torus is trivial and so the above construction applied to a flat metric on a torus would give Bianchi type I metrics.) Instead it is necessary to construct the appropriate circle bundle by some other method. The type II NUT metric is, in the coordinate form given by Siklos [41],

$$
-\tilde{U}^{-1} d t^{2}+(2 l)^{2} \tilde{U}(d \psi+\theta d \phi)^{2}+\left(t^{2}+l^{2}\right)\left(d \theta^{2}+d \phi^{2}\right),
$$

which is of the form taken by the metrics arising from the above construction. What needs to be checked in order to verify that this metric really does arise from a circle bundle over the torus is that the locally defined 1-form $d \psi+\theta d \phi$ is the coordinate form of a globally defined $U(1)$ connection $A$. This can be arranged, as follows from Chern-Weil theory. (For information on the relevant part of this theory see e.g. [46, pp.114-121]. That reference talks about Hermitian line bundles rather than circle bundles but it is easy to see that the two things are equivalent.) In order that the one-form come from a globally defined connection it suffices that its exterior derivative should define a global smooth 2 -form and that this 2 -form should satisfy an 
integrality condition. In the present case the 2 -form is $d \theta \wedge d \phi$, which is globally defined and smooth on the torus obtained by identifying $\theta$ and $\phi$ periodically and the integrality condition can be arranged by an appropriate choice of the coordinate volume of this torus. The time variation of the metric can once again be accomodated by constant conformal rescalings of the metric on the torus and the use of a time-dependent $a$.

\section{Some uniqueness results for Taub-NUT space- times}

In this section we shall prove some results concerning the question of uniqueness of extensions of the maximal globally hyperbolic region of the TaubNUT space-time. Let us start by a construction which shows that uniqueness of solutions of the Einstein equations fails whenever a Cauchy horizon occurs. This is true even if analyticity conditions on the metric and on the space-time are imposed. Let then $(M, g)$ be a vacuum extension of a maximal globally hyperbolic space-time $\left(M_{0}, g_{0}\right), M_{0} \subset M$, with Cauchy surface $\Sigma \subset M_{0}, \Sigma$ being a partial Cauchy surface in $M$. Let $\mathcal{B}$ be any embedded two-sided three-dimensional submanifold of $M \backslash \overline{\mathcal{D}(\Sigma ; M)}$. We shall moreover suppose that $M \backslash \overline{\mathcal{B}}$ is connected. Let $\left(M_{a}, g_{a}\right), a=1,2$, be two copies of $M \backslash \overline{\mathcal{B}}$ with the metric induced from $g$. As $\mathcal{B}$ is two-sided, there exists an open neighbourhood $\mathcal{O}$ of $\mathcal{B}$ such that $\mathcal{B}$ separates $\mathcal{O}$ into two disjoint open sets $\mathcal{O}_{a}, a=1,2$, with $\overline{\mathcal{O}_{1}} \cap \overline{\mathcal{O}_{2}}=\overline{\mathcal{B}}, \mathcal{O}_{1} \cap \mathcal{O}_{2}=\emptyset$. Let $\psi_{a}$ denote the natural embedding of $\mathcal{O}_{a}$ into $M_{a}$. Let $M_{3}$ be the disjoint union of $M_{1}, M_{2}$ and $\mathcal{O}$, with the following identifications: a point $p \in \mathcal{O}_{a} \subset \mathcal{O}$ is identified with $\psi_{a}(p) \in M_{a}$. It is easily seen that $M_{3}$ so defined is Hausdorff.

We can equip $M_{3}$ with the obvious manifold structure and an obvious metric $g_{3}$ coming from $\left(M_{1}, g_{1}\right),\left(M, g_{2}\right)$ and $\left(\mathcal{O},\left.g\right|_{\mathcal{O}}\right)$. Note that if $(M, g)$ were real analytic, then $\left(M_{3}, g_{3}\right)$ can be equipped with the obvious real analytic structure, with $g_{3}$ - real analytic with respect to this structure. Let finally $\left(M_{4}, g_{4}\right)$ be any maximal $\square$ vacuum (real analytic, if appropriate) extension of $\left(M_{3}, g_{3}\right)$. Then $\left(M_{4}, g_{4}\right)$ is a maximal vacuum (perhaps real analytic) extension of $\left(M_{0}, g_{0}\right)$ which clearly is not isometric to $(M, g)$.

The above allows one to construct many non-isometric maximal vacuum (perhaps analytic) extensions of a given extendable maximal globally hyperbolic space-time. Those extensions all have the property that the resulting metric "locally looks the same in all extensions", the lack of isometries coming from cut-and-paste games inflicted upon the original extension. Intuitively speaking, two sufficiently nearby observers living in two such extensions which originate from the globally byperbolic region will "see the

\footnotetext{
${ }^{17}$ cf., e.g., [5, Appendix C] for a proof of existence of space-times maximal with respect to some property. It should be pointed out that there is an error in that proof, as the relation $\prec$ defined there is not a partial order. This is however easily corrected by adding the requirement that the isometry $\Phi$ considered there restricted to some fixed threedimensional hypersurface be the identity.
} 
same metric". For this reason it seems of interest to present an alternative construction in which this will not be the case. The construction that follows will, however, not preserve analyticity of the metric if the original metric is analytic.

Consider thus $\left(M_{0}, g_{0}\right),(M, g), \Sigma$, as before, and let $\mathcal{N}_{a}, a=1,2$, be two smooth, embedded, null hypersurfaces in $M \backslash \mathcal{D}(\Sigma ; M)$ intersecting transversally along a smooth two-dimensional submanifold $S: \overline{\mathcal{N}_{1}} \cap \overline{\mathcal{N}_{2}}=S$, $\mathcal{N}_{1} \cap \mathcal{N}_{2}=\emptyset$. Choosing the $\mathcal{N}_{a}$ 's "small enough" we ensure that all the conditions in 38] needed for the well-posedness of the characteristic initial value problem in this setting are met. Let $\phi_{a}, a=1,2$, denote the free characteristic initial data induced from $g$ on $\mathcal{N}_{a}$ as described in [38], let $\psi$ denote the corresponding data on $S$, cf. [38] for details. "Making the $\mathcal{N}_{a}$ 's smaller" if necessary there exists on open embedded submanifold $\mathcal{O}$ of $M \backslash \mathcal{D}(\Sigma ; M)$ such that the metric $\left.g\right|_{\mathcal{O}}$ is uniquely determined by $\left(\phi_{a}, \psi\right)$. Note that this is true regardless of any global causality violations in $(M, g)$, as any Lorentzian metric is causally well behaved on "sufficiently small" regions.

Consider now a set of free charateristic initial data $\left(\tilde{\phi}_{1}, \phi_{2}, \psi\right)$, with $\tilde{\phi}_{1} \neq \phi_{1}$ but $\tilde{\phi}_{1}-\phi_{1}$ vanishing to infinite order on $\overline{\mathcal{N}_{1}}$ near $S$. Passing to subsets of the $\mathcal{N}_{a}$ 's and $\mathcal{O}$ if necessary there exists a solution $\tilde{g}$ of the vacuum characteristic initial value problem in $\mathcal{O}$ with initial data $\left(\tilde{\phi}_{1}, \phi_{2}, \psi\right)$ such that $g-\tilde{g}$ vanishes to infinite order on $\overline{\mathcal{O}}$ near $\mathcal{N}_{2}$. Let now $\left(M_{1}, g_{1}\right)$ be obtained by glueing $\left(M \backslash \mathcal{O},\left.g\right|_{M \backslash \mathcal{O}}\right)$ with $(\mathcal{O}, \tilde{g})$ along $\mathcal{N}_{2}$ in the obvious way. The desired extension of $\left(M_{0}, g_{0}\right)$ is obtained by taking any maximal vacuum extension of $\left(M_{1}, g_{1}\right)$.

The above constructions show that uniqueness of solutions of the Cauchy problem for the vacuum Einstein equations is lost whenever Cauchy horizons occur, and the best one can hope for is at most some form of uniqueness-upto-boundary of such extensions 18 . We shall show that such a result is true for Taub-NUT space-times. Before doing that some information about the isometry group of Taub-NUT space-times will be needed. Let us define the Taub space-time as $M=\left(t_{-}, t_{+}\right) \times L(p, 1)$, where $L(p, 1)$ is a lens space, with the metric

$$
d s=U^{-1} d t^{2}-(2 L)^{2} U(d \mu+\cos \theta d \varphi)^{2}-\left(t^{2}+L^{2}\right)\left(d \theta^{2}+\sin ^{2} \theta d \varphi^{2}\right) .
$$

Here $\mu, \theta, \varphi$ are Euler coordinates on $S^{3}$ (with some suitable supplementary identifications for the $L(p, 1)$ 's with $p \neq 1$ ). $U$ is given by eq. (2.35), and the $t_{ \pm}$'s are zeros of $U$. Note that if $m=0$, then the hypersurface $\Sigma_{0}=\{t=0\}$ is totally geodesic; let us in that case denote by $T$ the isometry which is a timereflection across $\Sigma_{0}$. By considerations involving uniqueness of maximal surfaces similar to those in 8 it is easily seen that for $m \neq 0$ no timeorientation-reversing isometries of $g$ exist.

\footnotetext{
${ }^{18}$ That is, there can be no uniqueness beyond Cauchy horizons unless some further conditions on the extensions are imposed. The problem is, that we do not know any reasonable conditions which would ensure either existence or at least uniqueness of extensions beyond Cauchy horizons.
} 
Recall, finally, that the map from $\mathbf{R}^{4}$ to itself defined by $(x, y, z, w) \rightarrow$ $(-x,-y, z, w)$ induces an analytic map from $L(p, 1)$ into itself. In the Euler coordinates this map takes the form $(\mu, \theta, \varphi) \rightarrow(-\mu, \theta,-\varphi)$. This leads obviously to an isometry of $M$ into itself, which we shall denote by $S$.

Let $G$ be the group of all isometries of $(M, g)$, let $G_{0}$ be the connected component of the identity of $G$. It is well known that $G_{0}$ acts transitively on each surface $\Sigma_{\tau}=\{t=\tau\}$. Moreover the isotropy group of each $p \in \Sigma_{\tau}$ consists of rotations in the planes perpendicular to the planes spanned by $\frac{\partial}{\partial \mu}$ and $\frac{\partial}{\partial t}$, in the coordinate system above. For $a, b= \pm$ let $\left(M^{a b}, g^{a b}\right)$ denote the Taub-NUT space-times described in [8]. We have the following:

Proposition 5.1 1. Let $G$ be the group of all isometries of a Taub spacetime $(M, g)$. If $m=0$, then $G$ has precisely four connected components, $G_{0}, T G_{0}, S G_{0}$ and $S T G_{0}$. If $m \neq 0$, then $G$ has precisely two connected components, $G_{0}$ and $S G_{0}$.

2. Let $G$ be the group of all isometries of a standard Taub-NUT spacetime $\left(M^{a b}, g^{a b}\right)$ :

If $m=0$ and $a b=+$, then $G$ has precisely two connected components, $G_{0}$ and $S T G_{0}$.

If $m=0$ and $a b=-$, then $G$ has precisely two connected components, $G_{0}$ and $T G_{0}$.

If $m \neq 0$, then $G$ has precisely one connected component, $G_{0}$, regardless of the value of $a b$.

Proof: 1. Let $\phi$ be an isometry of $(M, g)$. By composing $\phi$ with $T$ if necessary we may assume that $\phi$ preserves time-orientation. By Lemma 3.4 of [8] $\phi$ leaves the distribution $P$ of planes spanned by $\frac{\partial}{\partial t}$ and $\frac{\partial}{\partial \mu}$ invariant. By composing $\phi$ with $S$ if necessary we may assume that $\phi$ preserves the orientation of those planes.

Consider any $p \in \Sigma_{t}$, there exists $g \in G_{0}$ such that $(g \phi)(p)=p$. Because $g \phi$ is an isometry that preserves the distribution $P$, it must also preserve the distribution $P^{\perp}$ of planes perpendicular to the planes $P$. Replacing $g$ by $h g$, where $h \in G_{0}$ is an element of the isotropy group of $p$, we can achieve $\left.g \phi\right|_{P_{p}^{\perp}}=\left.i d\right|_{P_{p}^{\perp}}$. Now $g \phi$ preserves the hypersurfaces of homogeneity $\Sigma_{t}$, hence $(g \phi)^{*} \frac{\partial}{\partial t} \sim \frac{\partial}{\partial t}$; in fact $(g \phi)^{*} \frac{\partial}{\partial t}=\frac{\partial}{\partial t}$. As $g \phi$ is a Lorentz transformation of $P_{p}$ into itself which preserves orientation, we must have $\left.g \phi\right|_{P_{p}}=i d_{P_{p}}$. We have thus shown that $\left.g \phi\right|_{T_{p} M}=i d_{T_{p} M}$, which together with $(g \phi)(p)=p$ and standard results on isometries shows that $g \phi=i d$.

2. Let $\phi$ be an isometry of $\left(M^{a b}, g^{a b}\right)$ into itself. By Proposition 2.1 of [8] $\left.\phi\right|_{M}$ is an isometry of the Taub space $(M, g) \subset\left(M^{a b}, g^{a b}\right)$ into itself. The extendability of isometries of the Taub region in $S T G_{0}$ to isometries of $\left(M^{a b}, g^{a b}\right)$ with $a b=+$ follows now from construction and from eq. (8c) in [8], similarly for isometries in $T G_{0}$ in the $a b=-$ case. The nonextendability 
of the appropriate isometries in the remaining cases follows by considerations similar to the proof of Theorem 3.1 of [8].

Proof of Theorem 1.2: By proposition 4.4 for any $p \in \partial \mathcal{D}(\Sigma)$ the orbits of the extensions of the Killing vector fields from $\stackrel{\circ}{\mathcal{D}}(\Sigma)$ to $\partial \mathcal{D}(\Sigma)$ are threedimensional. It follows that the arguments of the proof of Lemma 2.5 apply for any $p \in \partial \mathcal{D}(\Sigma)$, so that for any $p \in \partial \mathcal{D}(\Sigma)$ there exists a neighbourhood $\mathcal{W}_{p}$ in $\overline{\mathcal{D}(\Sigma)}$ of $p$ and an equivariant one-to-one isometry $i_{p}$ of $\mathcal{W}_{p}$ into a subset of a standard Taub-NUT space time $\left(M^{a b}, g^{a b}\right)$. Let us show that the $i_{p}$ 's can be patched together to a single isometry on every connected component of the Cauchy horizon, say $i^{+}$on $\mathcal{H}^{+}$, and $i^{-}$on $\mathcal{H}^{-}$. Let $p$ be a point on the future Cauchy horizon. Consider the set of pairs $(\mathcal{W}, \phi)$ where $\mathcal{W}$ is an open neighbourhood of $p$ in $\overline{\mathcal{D}(\Sigma)} \cap I^{+}(\Sigma)$ and $\phi$ is an isometry of $\mathcal{W}$ onto an open subset of a Taub-NUT space time. Now fix one of these pairs, say $\left(\mathcal{W}_{0}, \phi_{0}\right)$, and restrict consideration to those pairs $(\mathcal{W}, \phi)$ which agree with $\left(\mathcal{W}_{0}, \phi_{0}\right)$ on a neighbourhood of $p$. Any two isometries of this type agree on the intersection of their domains and so there exists a maximal element $\left(\mathcal{W}_{\max }, \phi_{\max }\right)$ in this class. $\mathcal{W}_{\max }$ is by definition open. Let $q$ be a point of its boundary. There exists a neighbourhood $\mathcal{Z}$ of $q$ and an isometry $\psi$ of $\mathcal{Z}$ onto a subset of Taub-NUT space time by Lemma 2.5. Let $r$ be a point of the horizon in $\mathcal{W}_{\max } \cap \mathcal{Z}$. By composing with an isometry of Taub-NUT spacetime it can be arranged that $\psi(r)=\phi_{\max }(r)$. The construction of the local isometry in Lemma 2.5 also includes a null frame and the vectors $l$ and $n$ in this frame are uniquely defined. Moreover $m$ is defined up to multiplication by $e^{i \theta}, \theta$ constant. Let $\left(l^{\prime}, n^{\prime}, m^{\prime}\right)$ and $\left(l^{\prime \prime}, n^{\prime \prime}, m^{\prime \prime}\right)$ be the images of $(l, n, m)$ under $\phi_{\max }$ and $\psi$ respectively. The spin coefficients of $\left(l^{\prime}, n^{\prime}, m^{\prime}\right)$ have by construction a special form and those of $\left(l^{\prime \prime}, n^{\prime \prime}, m^{\prime \prime}\right)$ are equal to those of $\left(l^{\prime}, n^{\prime}, m^{\prime}\right)$. It follows that $l^{\prime \prime}=l^{\prime}, n^{\prime \prime}=n^{\prime}$ and $m^{\prime \prime}=e^{i \theta} m^{\prime}$ for a constant $\theta$. By composing $\psi$ with an isometry of Taub-NUT space time which fixes $\psi(r)$ it is possible to set $m^{\prime \prime}=m^{\prime}$. After this has been done $\phi_{\max }$ and $\psi$ agree on a neighbourhood of $r$ and hence together they define an isometry on $\mathcal{W}_{\max } \cup \mathcal{Z}$, contradicting the definition of $\mathcal{W}_{\max }$. Hence $\mathcal{W}_{\max }$ has no boundary point and, since the future Cauchy horizon is connected, $\mathcal{W}_{\max }=\overline{D(\Sigma)} \cap I^{+}(\Sigma)$. By equivariance the $i^{ \pm}$'s can be extended to a oneto-one isometry, still denoted by $i^{ \pm}$, from $\mathcal{D}(\Sigma, M)$ into $\left(M^{a b}, g^{a b}\right)$. If $\mathcal{H}^{+}$or $\mathcal{H}^{-}$is empty we are done, if not, consider the map $j=i^{+} \circ\left(i^{-}\right)^{-1}$ which is an isometry from the Taub region of $\left(M^{a b}, g^{a b}\right)$ into itself. Without loss of generality we may assume that $j$ preserves time orientation. If $j \in G_{0}$ then the isometries $i^{t}$ and $i^{-}$can be patched together to a one-to-one isometryup-to boundary of $\overline{\mathcal{D}(\Sigma, M)}$ into $\left(M^{++}, g^{++}\right)$. Similary if $j \in S G_{0}$, then $i^{+}$ and $i^{-}$can be patched together to a one-to-one isometry up-to-boundary of $\overline{\mathcal{D}(\Sigma, M)}$ into $\left(M^{+-}, g^{+-}\right)$, and the result follows.

Our next result proves uniqueness of the standard Taub-NUT space-times, in the class of maximal space-times with an action of $G=S U(2)$ or $S O(3)$ by isometries:

Theorem 5.2 Let $(M, g)$ be a vacuum Bianchi IX space-time with a com- 
pact partial Cauchy surface $\Sigma$ and nontrivial Cauchy horizon, $\partial \mathcal{D}(\Sigma) \neq \emptyset$. Suppose, moreover, that $(M, g)$ is maximal 11 in the class of vacuum spacetimes on which $G=S U(2)$ or $S O(3)$ acts by isometries. Then $(M, g)$ is a standard Taub-NUT space-time.

Proof: Let $\Sigma$ be a partial Cauchy surface in $M$, by Theorem 1.2 there exists a one-to-one map $i$ which maps $\overline{\mathcal{D}(\Sigma ; M)}$ into $\overline{\mathcal{D}\left(i(\Sigma), M^{\prime}\right)}$, and which is a

smooth isometric diffeomorphism between $\stackrel{\circ}{\mathcal{D}}(\Sigma ; M)$ and $\stackrel{\circ}{\mathcal{D}}\left(i(\Sigma), M^{\prime}\right)$. Here $M^{\prime}$ is the corresponding standard Taub-NUT space-time. By maximality and homogeneity $i$ has to be onto. The result follows by a Siklos tetrad construction argument, as in Section 2, and by simple maximality arguments.

For other Bianchi types standard extensions can be constructed for each extendible Bianchi model, as should be clear for the explicit form of the metrics given in [42, 41] or as discussed in Section 1. Then the obvious equivalents of Theorems 1.2 and 5.2 hold, the details are left to the reader. Note that it follows from Lemma 4.2 that future and past Cauchy horizons occur simultaneously only for the Bianchi IX models, so that the equivalent of Proposition 5.1 is not needed in the non-Bianchi-IX cases.

Let us finally mention that uniqueness of standard extensions of KantowskiSachs models does not follow immediately from what was said above, though we believe that the arguments given could be adapted to obtain such results. We have, however, not made attempts to analyze this problem.

\section{References}

[1] B. Berger, P.T. Chruściel, and V. Moncrief, On asymptotically flat space-times with $G_{2}$ invariant Cauchy surfaces, MPA preprint $\mathbf{7 9 7}$ (1994), gr-qc/9404005, Annals of Physics, in press.

[2] R. Budic, J. Isenberg, L. Lindblom, and P. Yasskin, On the determination of Cauchy surfaces from intrinsic properties, Commun. Math. Phys. 61 (1978), 87-95.

[3] Y. Choquet-Bruhat and J. York, The Cauchy problem, General Relativity (A. Held, ed.), Plenum Press, New York, 1980.

[4] F. Christiansen, H.H. Rugh, and S.E. Rugh, Painlevé integrability and periodic orbits for the mixmaster universe, 1994, preprint, solvint/9406002.

[5] P.T. Chruściel, On uniqueness in the large of solutions of Einstein equations ("Strong Cosmic Censorship"), Australian National University Press, Canberra, 1991.

[6] _ On uniqueness in the large of solutions of Einstein equations ("Strong Cosmic Censorship"), Cont. Math. 132 (1992), 235-273. 
[7] _ On completeness of orbits of Killing vector fields, Class. Quantum Grav. 10 (1993), 2091-2101.

[8] P.T. Chruściel and J. Isenberg, Non-isometric vacuum extensions of vacuum maximal globally hyperbolic space-times, Phys. Rev. D48 (1993), 1616-1628.

[9] P.T. Chruściel, J. Isenberg, and V. Moncrief, Strong cosmic censorship in polarized Gowdy space-times, Class. Quantum Grav. 7 (1990), 16711680.

[10] C.B. Collins and G.F.R. Ellis, Singularities in Bianchi cosmologies, Phys. Rep. 56 (1979), 65-105.

[11] G. Contopoulos, B. Grammaticos, and A. Ramani, Painlevé analysis for the Mixmaster universe model, Jour. Phys. A: Math. Gen. 26 (1993), 5795-5799.

[12] _ Integrability of the Mixmaster universe, Deterministic Chaos in General Relativity (D. Hobill, A. Burd, and A. Coley, eds.), Plenum Publishing, New York, 1994, (Proc. of the Kananaskis Conference on chaos in general relativity, July 1993).

[13] J. Ehlers and W. Kundt, Exact solutions of the gravitational field equations, Introduction to current research (L. Witten, ed.), J. Wiley \& Sons, New York, 1962, pp. 49-101.

[14] H. Federer, Geometric measure theory, Springer Verlag, New York, 1969, (Die Grundlehren der mathematischen Wissenschaften, Vol. 153).

[15] A. Fischer, The theory of superspace, Relativity (M. Carmeli, S. Fickler, and L. Witten, eds.), Plenum Press, New York, 1970.

[16] J. Foster, DS-spaces of Robinson \& Trautman: Topological identifications and asymptotic symmetry, Proc. Cambridge Phil. Soc. 66 (1969), $521-531$.

[17] Y. Fujiwara, H. Ishihara, and H. Kodama, Comments on closed Bianchi models, Class. Quantum Grav. 10 (1993), 859-867.

[18] G. Galloway, Some results on Cauchy surface criteria in Lorentzian geometry, Illinois Jour. Math. 29 (1985), 1-10.

[19] B. Grubišić and V. Moncrief, The Mixmaster spacetime, Geroch's transformation, and constants of motion, Phys. Rev. D49 (1994), 2792-2800.

[20] G.S. Hall, The global extension of local symmetries in general relativity, Class. Quantum Grav. 6 (1989), 157-161.

[21] S. Hawking and G.F.R. Ellis, The large scale structure of space-time, Cambridge University Press, Cambridge, 1973. 
[22] D. Hobill, A brief review of "deterministic chaos in general relativity", Deterministic Chaos in General Relativity (D. Hobill, A. Burd, and A. Coley, eds.), Plenum Publishing, New York, 1994, (Proc. of the Kananaskis Conference on chaos in general relativity, July 1993).

[23] J. Isenberg, Progress on strong cosmic censorship, Cont. Math. 132 (1992), 403-418.

[24] J. Isenberg and M. Jackson, Ricci flow of locally homogeneous geometries on closed manifolds, Jour. Diff. Geom. 35 (1992), 723-741.

[25] J. Isenberg and V. Moncrief, Symmetries of cosmological Cauchy horizons with exceptional orbits, Jour. Math. Phys. 26 (1985), 1024-1027.

[26] _ Asymptotic behavior of the gravitational field and the nature of singularities in Gowdy space-times, Ann. of Phys. (NY) 199 (1990), $84-122$.

[27] T. Koike, M. Tanimoto, and A. Hosoya, Compact homogeneous universes, Jour. Math. Phys. 35 (1994), 4855-4888.

[28] M. MacCallum, The mathematics of anisotropic spatially-homogeneous cosmologies, Physics of the expanding universe (M. Demiański, ed.), Springer Lecture Notes in Physics 109, 1979, Cracow School on Cosmology, Jodłowy Dwór, September 1978, pp. 1-59.

[29] G. Mess, Lorentz space-times of constant curvature, I.H.E.S. preprint IHES/M/90/28, 1990.

[30] C.W. Misner, Taub-NUT space as a counterexample to almost anything, Relativity Theory and Astrophysics (Providence, Rhode Island), AMS, Providence, Rhode Island, 1967, Lectures in Appl. Math., vol. 8, pp. 160-169.

[31] V. Moncrief and D. Eardley, The global existence problem and cosmic censorship in general relativity, Gen. Rel. Grav. 13 (1981), 887-892.

[32] E.T. Newman and K.P. Tod, Asymptotically flat space-times, General Relativity and Gravitation (A. Held, ed.), Plenum, New York and London, 1980, pp. 1-36.

[33] K. Nomizu, On local and global existence of Killing vector fields, Ann. Math. 72 (1960), 105-120.

[34] R. Penrose, Gravitational collapse - the role of general relativity, Riv. del Nuovo Cim. 1 (numero speziale) (1969), 252-276.

[35] — Techniques of differential topology in relativity, SIAM, Philadelphia, 1972, (Regional Conf. Series in Appl. Math., vol. 7). 
[36] A.D. Rendall, Global properties of locally spatially homogeneous cosmological models with matter, preprint gr-qc/9409009, Math. Proc. Camb. Phil. Soc., in press.

[37] A.D. Rendall, Cosmic censorship for some spatially homogeneous cosmological models, Ann. Phys. 233 (1994), 82-96.

[38] A.D. Rendall, Reduction of the characteristic initial value problem to the Cauchy problem and its applications to the Einstein equations, Proc. Roy. Soc. London A 427 (1990), 221-239.

[39] M.P. Ryan and L.C. Shepley, Homogeneous relativistic cosmologies, Princeton University Press, Princeton, 1975.

[40] P. Scott, The geometries of 3-manifolds, Bull. London Math. Soc. 15 (1983), 401-487.

[41] S.T.C. Siklos, Two completely singularity-free NUT spacetimes, Phys. Lett. A 59 (1976), 173-174.

[42] _ Occurrence of whimper singularities, Commun. Math. Phys. 58 (1978), 255-272.

[43] I.M. Singer, Infinitesimally homogeneous spaces, Commun. Pure Appl. Math. XIII (1960), 685-697.

[44] W. Thurston, Three dimensional manifolds, Kleinian groups and hyperbolic geometry, Bull. of the AMS 6 (1982), 357-381.

[45] R.M. Wald, General relativity, University of Chicago Press, Chicago, 1984.

[46] N. Woodhoouse, Geometric quantization, Clarendon Press, Oxford, 1980. 